\title{
Evidence for RNA or protein transport from somatic tissues to the male reproductive tract in mouse
}

\author{
Vera Rinaldi ${ }^{1}$, Kathleen Messemer ${ }^{2,3}$, Kathleen Desevin ${ }^{1}$, Fengyun Sun ${ }^{1}$, Bethany C. \\ Berry $^{1}$, Shweta Kukreja1 ${ }^{1}$, Andrew R. Tapper ${ }^{4,5}$, Amy J. Wagers ${ }^{2,3,6}$, and Oliver J. \\ Rando ${ }^{1 \dagger}$
}

${ }^{1}$ Department of Biochemistry and Molecular Biotechnology, University of Massachusetts Chan Medical School, Worcester, MA 01605, USA

2Section on Islet Cell and Regenerative Biology, Joslin Diabetes Center, Boston, MA 02215, USA

${ }^{3}$ Department of Stem Cell and Regenerative Biology, Harvard University, and Harvard Stem Cell Institute, Cambridge, MA 02138, USA

${ }^{4}$ Department of Neurobiology, University of Massachusetts Chan Medical School, Worcester, MA 01605, USA

${ }^{5}$ Brudnick Neuropsychiatric Research Institute, University of Massachusetts Chan Medical School, Worcester, MA 01605, USA

${ }^{6}$ Paul F. Glenn Center for the Biology of Aging, Harvard Medical School, Boston, MA USA

† To whom correspondence should be addressed. Email:

Oliver.Rando@umassmed.edu

\begin{abstract}
The development of tools to manipulate the mouse genome, including knockout and transgenic technology, revolutionized our ability to explore gene function in mammals. Moreover, for genes that are expressed in multiple tissues or at multiple stages of development, the use of tissue-specific expression of the Cre recombinase allows gene function to be perturbed in specific cell types and/or at specific times. However, it is well known that putative tissue-specific promoters often drive unanticipated "off target" expression. In our efforts to explore the biology of the male reproductive tract, we unexpectedly found that expression of Cre in the central nervous system resulted in recombination in the epididymis, a tissue where sperm mature for $\sim 1-2$ weeks following the completion of testicular development. Remarkably, we not only observed reporter expression in the epididymis when Cre expression was driven from neuron-specific transgenes, but also when Cre expression in the brain was induced from an AAV vector carrying a Cre expression construct. A surprisingly wide range of Cre drivers - including six different neuronal promoters as well as the adipose-specific AdipoQ Cre promoter exhibited off target recombination in the epididymis, with a subset of drivers also exhibiting unexpected activity in other tissues such as the reproductive accessory glands. Finally, using a combination of parabiosis and serum transfer experiments, we find evidence supporting the hypothesis that Cre may be trafficked from its cell of origin
\end{abstract}


to the epididymis through the circulatory system. Together, our findings should motivate extreme caution when interpreting conditional alleles, and suggest the exciting possibility of inter-tissue RNA or protein trafficking in modulation of reproductive biology.

\section{INTRODUCTION}

The transition from unicellular life to multicellular life allowed organisms to delegate tasks to cells which could then specialize rather than being required to carry out all the functions of the organism. Functions of different cell types are coordinated by a wide array of mechanisms, including direct cell-cell contacts within a tissue, shared access to nutrient pools, and both local (paracrine) and longer-range trafficking of small molecule and peptide signals. These signals often carry information about organismal states such as hunger/satiety (insulin, leptin, etc.), reproductive status (testosterone, estrogen, etc.), stress (cortisol, adrenaline, etc.), and a multitude of other relatively blunt summaries of organismal status.

In addition to such coarse-grained signals of organismal physiology, it is increasingly understood that more detailed molecular signals can be communicated between distant tissues. This is particularly clear in the well-documented cases of systemic RNA trafficking seen in worms and plants. For instance, in C. elegans, introduction of double stranded RNA either by injection or by feeding with dsRNAexpressing bacteria leads to organism-wide silencing of homologous genes [1, 2], demonstrating that RNAs can traffic from the gut or hypodermis throughout the body, with sequence-specific regulatory consequences in the recipient tissue. Moreover, expression of RNAi triggers in neurons has been shown to silence target genes in the germline [3], again demonstrating trafficking of relatively precise RNA signals between tissues. Systemic RNA trafficking is also well-described in plants, as various means of introducing dsRNA into aerial tissues can drive sequence-specific silencing in roots [4, 5]. These examples of systemic RNA trafficking highlight the potential for more precise molecular signaling between tissues than achievable via the relatively blunt information content available with hormonal signals.

In mammals, many studies have reported RNA or protein trafficking between different cell types within the same tissue [6], or between distant tissues [7, 8]. Although this is well documented for several somatic tissues, in the unique case of the germline there are only sporadic reports of RNA trafficking from distant tissues to germ cells themselves [9-11]. That said, it is worth noting that gametogenesis is supported by a wide variety of somatic cells and accessory tissues, and these could serve as intermediaries between distal tissues and developing gametes. These reproductive support cells/tissues include testicular cell populations such as the Sertoli cells that act as the spermatogonial stem cell niche and broadly support testicular spermatogenesis, as well as other tissues such as the epididymis, seminal vesicle, and prostate that contribute to sperm maturation and delivery to the female reproductive tract. Given the key roles of these tissues in sperm development and maturation, we were motivated to explore the potential for inter-tissue signaling to play a role in modulating reproductive tract physiology. 
Here, we leverage the exquisite sensitivity of Cre-dependent recombination to identify inter-tissue communication pathways in the adult mouse, focusing on the male reproductive tract. We find that expression of Cre in multiple distant tissues, most notably including the central nervous system (CNS), results in expression of a Credependent reporter gene in the epididymis. Reporter activity is observed following Cre expression in the CNS induced via adeno-associated viral (AAV) transduction, as well as in animals carrying Cre under the control of a variety of tissue-specific promoters. Parabiosis (in which two animals share a conjoined circulatory system) and serum transfer studies suggest that this "off target" reporter activity likely results from Cre trafficking from distant tissues to the epididymis via the circulatory system. Together, our findings highlight the epididymis as a surprisingly common site for unanticipated off target effects in conditional KO studies, and establish a novel model for inter-tissue communication in mammals.

\section{RESULTS}

Throughout this study, we utilize the Ai14D reporter mouse strain $[12,13]$ to assay Cre activity throughout the body (Figure 1A). This reporter carries a transgene at the "safe harbor" Rosa26 locus, with a ubiquitously-expressed CAG promoter driving expression of a transgene with a 3X STOP cassette flanked by two LoxP sites, followed by a fluorescent reporter gene encoding tdTomato. Cre recombinase excises the STOP cassette, resulting in robust tdTomato (hereafter, Tomato) expression in Cre-expressing cells. For the majority of the experiments described below, Tomato expression is assayed in tissues cleared using a modified CLARITY [14, 15] protocol, and visualized using light sheet microscopy to survey entire tissues. Figure 1B shows a typical negative control image of an epididymis taken from an Ai14D animal lacking any Cre expression. The larger panel includes the caput, corpus, and cauda epididymis along with a large section of the vas deferens, while smaller inset images show representative cross-sections of the indicated regions of the epididymis.

As a positive control, we crossed the Ai14D animal to a mouse strain bearing the epididymis-specific Defb41 iCre driver [16]. Figure 1C shows the anticipated Tomato expression in these double-transgenic animals. Consistent with the distribution of Defb41 mRNA expression observed in surveys of epididymal gene expression [17, 18], we find that Defb41icre drives reporter expression in a highly localized manner in the proximal (caput) epididymis. This segment-delimited expression is readily apparent in images of the whole epididymis, while cross-sections show that Tomato expression is confined to the principle cells (but not basal or other interstitial cells) of the epididymal epithelium (Figure 1C, Supplemental Figure 1). Tomato expression is notably absent from the sperm-filled lumen. Together, these images provide representative positive and negative controls illustrating the utility of the Ai14D strain as a sensitive and specific reporter for Cre activity.

\section{Neuronal Cre expression results in reporter activity in the cauda epididymis}

Motivated by observations in $C$. elegans that small RNA expression in neurons can result in siRNA activity in the germline [3], we set out to explore the potential for neuronal Cre expression in mice to drive cell non-autonomous Cre activity elsewhere in 
the body. In two series of experiments, Cre expression was induced in the mouse brain via stereotaxic delivery of Adeno-Associated Virus (AAV) constructs engineered to drive Cre expression in transduced cells (Figure 2A). In an initial series of experiments, we utilized an AAV2-retro-Ef1a-Cre construct introduced into the prefrontal cortex (Supplemental Figure S2), while follow-up studies targeted the lateral somatosensory cortex, caudate putamen, and nucleus accumbens using an AAV9-pCMV-Cre construct (Figure 2B). Remarkably, examination of the epididymis in both cases revealed robust Tomato expression in the cauda (distal) epididymis and in the vas deferens (Figure 2C). Tomato expression was reproducibly observed in all epididymis samples isolated from ten AAV-transduced animals, including three animals injected in the prefrontal cortex (Supplemental Figure S2) as well as seven animals injected in the lateral somatosensory cortex, caudate putamen, and nucleus accumbens (Figure 2C, Supplemental Figure S3). As in the case of the Defb41iCre driver, closer examination of our imaging data showed that Tomato expression was confined to the epididymal epithelium (inset, Figure 2C, Supplemental Figure S3), with no detectable expression in the sperm filling the epididymal lumen (see below).

\section{Neuronal Cre expression driven from transgenes also induces recombination in the epididymis}

An exciting potential explanation for the ability of neuronal Cre expression to drive recombination in the epididymis would be that Cre RNA or protein synthesized in neurons is somehow trafficked to the male reproductive tract. However, the use of viral transduction to induce Cre expression leaves open the possibility that viral particles entering the circulation could plausibly exhibit unanticipated tropism for the epididymis.

We therefore turned to an orthogonal method for driving Cre expression in neuronal subpopulations by crossing Ai14D mice to various driver lines expressing Cre under the control of cell type-specific promoters (Figure 3A). We illustrate this approach using Vgat Cre mice that express Cre under the control of the vesicular GABA transporter (S/c32a1, or Vgat) promoter, which drives Cre expression in inhibitory GABAergic neurons in the central nervous system. Consistent with expectations, we observed widespread Tomato expression in the brain of double transgenic animals, with no detectable expression in the liver, kidney, intestine, or testis (Figure 3B). Remarkably, similar to the reporter expression observed following viral induction of neuronal Cre expression (Figure 2), Cre expression from the Vgat transgene also resulted in robust epididymal Tomato expression in Ai14D; Vgat ${ }^{\text {Cre }}$ double transgenic animals (Figure 3C). Closer inspection of double transgenic epididymis by microscopy revealed Tomato expression exclusively in the principal cells of the epididymal epithelium (Figure 3D), and we further confirmed the presence of Tomato-positive cells by FACS

(Supplemental Figure S4). Intriguingly, we observed patchy Tomato expression in any given cross section, suggesting either that a subset of principal cells are capable of receiving Cre shipments, or that Cre transfer is extremely inefficient and only a subset of cells are stochastically labeled.

Finally, we sought to determine whether Cre expression in reporter animals resulted in epididymal Tomato signal as a result of Cre itself being shipped from neurons to the epididymis, vs. Cre-induced Tomato produced in neurons being shipped to the epididymis. We therefore assayed recombination at the LoxP-STOP cassette in 
the epididymis, finding recombination at the reporter locus in genomic DNA isolated from the cauda epididymis (Figure 3E). These findings strongly support the hypothesis that Cre activity (RNA or protein), rather than Tomato itself, is the relevant molecular signal trafficked from the CNS to the epididymis.

Together, our findings suggest the possibility that Cre RNA or protein synthesized in the central nervous system is transported to the male reproductive tract, presumably through the circulation (see below). Importantly, the two experimental schemes used to drive Cre expression in the central nervous system have orthogonal potential artifacts. In the case of AAV transduction, the injected virus could conceivably enter the circulation and might have an unknown tropism for the epididymis. However, this concern does not apply to the use of transgenic constructs to drive Cre expression. Conversely, it is well-known that purportedly tissue-specific Cre drivers are often less cell type-specific than expected $[19,20]$, and the male reproductive tract - the testis in particular - is well known to express an unusually high fraction of the genome.

However, this cannot explain our results using viral transduction to drive Cre expression in the CNS. We further test the hypothesis that Cre is trafficked via the circulation to the male reproductive tract below.

\section{Many-to-many mapping between Cre drivers and recipient tissues}

Our findings thus far suggest the intriguing possibility that the epididymis might be a privileged recipient of neuronally-derived molecular cargo. To expand our survey of neuronal Cre lines beyond our initial studies using $\mathrm{Vgat}^{\mathrm{Cre}}$, we crossed the Ai14D line to a number of additional CNS Cre drivers: Fev (serotonergic neurons), Gad2 (GABAergic neurons), Syn1 (pan-neuronal), Nestin (pan-neuronal), and Drd1a (dopaminoceptive neurons), ChAT (cholinergic neurons), Dat1 (dopaminergic neurons), and Gfap (glia). We also explored two non-neuronal Cre drivers, crossing the Ai14D animal to Alb (liver) and Adiponectin (adipose tissue) Cre lines. We note that the majority of these genes are either undetectable or expressed at extremely low levels $(\sim 1 \mathrm{ppm})$ in the epididymis as assayed either by bulk or single cell RNA-Seq [18].

For each of these double transgenic lines, we surveyed a range of tissues, typically including brain, intestine, liver, lung, kidney, testis, seminal vesicle/prostate, and the epididymis. Images for key examples are documented in Figures 4-5 and Supplemental Figures S5-8. Overall, we find that the connection between Cre expression in neurons and reporter expression in the epididymis is not a one-to-one mapping, but rather many-to-many: not only do some non-neuronal Cre lines drive reporter expression in the epididymis, but many Cre lines also drive reporter expression in additional tissues such as the intestine or seminal vesicle. Findings of particular interest are detailed below.

Most notably, we find that a wide range of neuronal Cre lines drive Tomato expression in the epididymis. Focusing first on the putative "pan-neuronal" Cre lines, driven by the Synapsin or Nestin promoters, we found extensive Tomato staining throughout the epididymis in both double transgenic lines (Supplemental Figure S6). However, we noted that Tomato expression in the Syn Cre animals was not confined to the epididymal epithelium and instead filled the epididymis lumen (Supplemental Figure S6A), suggestive of Tomato-positive sperm. Indeed, offspring of the Ai14D; Syn Cre double transgenics exhibited Tomato expression throughout the body, consistent 
with prior reports of Syn Cre driving germline recombination [21]. Similarly, the Nestin Cre driver has also been reported to drive germline recombination [22, 23], and we did obtain Tomato-positive offspring of Ai14D; Nes ${ }^{\text {Cre }}$ double transgenics. That said, in contrast to the Ai14D; Syn Cre animals we found no detectable labeling of sperm in Ai14D; Nes ${ }^{C r e}$ animals, with Tomato expression in the epididymis largely confined to the epididymal epithelium (Supplemental Figure S6B). This suggests that $\mathrm{Nes}^{\mathrm{Cre}}$-mediated recombination occurs relatively late during spermatogenesis in germ cells, with recombination in the epididymal epithelium occurring independently - probably induced by the same CNS $\rightarrow$ epididymis trafficking seen for several other neuronal Cre drivers (Figure 3 and below).

Turning from pan-neuronal promoters to more cell type-specific CNS Cre drivers, we consistently observed Tomato expression in the epididymis induced when Cre was expressed from Vgat, Fev, Gad2, and Drd1a promoters (Figures 3-4). For two of these Cre drivers - Fev and Vgat - we find that Tomato expression was strongest in the cauda epididymis and vas deferens, similar to our findings obtained using AAV to drive Cre expression in the CNS. In contrast, both Ai14D; Gad2Cre and Ai14D; Drd1aCre double transgenic animals exhibited patchy epithelial staining throughout the epididymis (Figure 4). Remarkably, off-target epididymal recombination was not unique to neuronal Cre drivers, as we also observed robust Tomato expression and reporter locus recombination in animals carrying the adipose-specific AdipoQ Cre (Figure 5). In contrast, reporter animals carrying the liver-specific $A / b^{C r e}$ transgene exhibited barelydetectable Tomato expression in the epididymis with few Tomato-positive cells detected throughout the entire tissue - one or two cells per longitudinal section (Supplemental Figure S5) - indicating that not all Cre transgenes drive extensive recombination in the epididymis.

Although our initial studies suggested a privileged molecular trafficking pathway from the central nervous system to the male reproductive tract (Figures 2-4), the robust Tomato expression driven by the adipose-specific AdipoQ Cre driver (Figure 5) suggested the potential for additional avenues for unanticipated inter-tissue Cre trafficking. Not only did non-neuronal Cre drivers direct recombination in the epididymis, but multiple neuronal Cre drivers exhibited off target activities in tissues beyond the epididymis. Most notably, within the male reproductive tract we observed robust Tomato expression in the reproductive accessory glands of the Nes, Fev, and Gad2 lines (Supplemental Figures S7-8).

\section{Cre activity likely traffics through the circulation to target the epididymis}

Together, our findings reveal that the epididymis is a surprisingly common target of a variety of Cre drivers that are not generally thought to be expressed in the male reproductive tract. While the observations made using double transgenic lines could result from leaky expression of the promoters in question - whether in adulthood or during early development of the epididymis - this hypothesis would not explain the results observed following AAV-mediated Cre expression in the brain (Figure 2). Nonetheless, we sought to definitively test the hypothesis that Cre activity, whether protein or RNA, synthesized in distant tissues can make its way to the male reproductive tract through the circulation. 
As an initial test of this hypothesis, we generated parabiotic animal pairs [24], in which two animals are surgically joined (hereafter represented as animal1::animal2) to establish a conjoined circulatory system (Figure 6A, Supplemental Figures S9A-C). Here, parabioses were established between a Cre driver line (focusing on the Vgat and Nestin Cre lines) and an Ai14D reporter animal. Eight to nine weeks after establishing the parabiosis, we sacrificed the animal pairs and harvested tissues for analysis. We detected moderate Tomato expression in the epididymis of the Ai14D recipient animal in a parabiotic Ai14D::Vgat Cre pair (Figure 6B, Supplemental Figure S9B), along with a faint band supporting recombination at the reporter locus in one recipient (Figure 6C). However, in two other Ai14D animals we found no evidence for recombination by PCR (Supplemental Figure S9C), motivating more extensive characterization of the potential for Cre transfer through the circulation.

Given the technical and logistical challenges involved in generating large numbers of parabiotic animal pairs, we attempted to transfer any potential circulating Cre activity by injecting serum or circulating exosomes (enriched via tangential flow filtration - TFF [25]), from various Cre lines into Ai14D reporter animals (Figure 6D). In an initial experiment, we obtained serum from a VgatCre animal and transferred it into the tail vein of an Ai14D male. This procedure was repeated every other day, for a total of three transfers over five days. As shown in Figures 6E-F, this resulted in epididymal LoxP recombination and Tomato expression in animals receiving $V_{\text {gat }}{ }^{C r e}$ serum, while recipients of serum from FVB or $A / b^{C r e}$ did not express Tomato or exhibit recombination at the reporter locus.

Follow up studies were somewhat ambiguous (Supplemental Figure S9D-F). We initially set out to leverage the serum transfer approach to fractionate serum and identify the circulating material responsible for driving recombination in recipient animals. We found no Tomato expression in an Ai14D male that had received TFFenriched exosomes from nine $\mathrm{Vgat}^{\mathrm{Cre}}$ animals spread across three injection days (Supplemental Figure S9E, top right panel). Across many subsequent experiments, we used a range of donor animals carrying various Cre drivers and varied our injection scheme to focus on whole serum, concentrated exosomes, or combinations of the two. Ultimately, across over 20 recipient animals we documented clear evidence for recombination in six animals, with positive results in recipients of $\mathrm{Vgat}^{\mathrm{Cre}}$ serum, $\mathrm{Vgat}$ Cre serum and exosomes, Gad2 ${ }^{\mathrm{Cre}}$ serum and exosomes, a mixture of Gad2 ${ }^{\mathrm{Cre}}$ and Drd1Cre serum and exosomes, Nes ${ }^{\mathrm{Cre}}$ serum and exosomes, and AdipoQ ${ }^{\mathrm{Cre}}$ serum and exosomes (Supplemental Figures S9E-F). However, similar or nearly-identical injection schemes often resulted in no detectable Tomato expression or recombination. Although we attempted to control for variables ranging from the recipient animal's age, to injection time during the day, we have not been able to identify variables that reliably distinguish injection schema that lead to recombination from those that fail.

Taken together, our data support the hypothesis that off target Cre activity in the epididymis is likely mediated by Cre trafficking through the circulation, although the difficulty replicating this transfer reliably impedes further mechanistic follow-up.

\section{DISCUSSION}

Our observations reveal the epididymis as a surprisingly common "off target" tissue for a variety of Cre drivers that are not believed to be expressed in the male reproductive 
tract. We find a many-to-many mapping, with "sender" tissues including multiple neuronal subpopulations along with adipose tissue, and recipient tissues including the epididymis, seminal vesicle, prostate, and intestine. Importantly, different Cre lines drive recombination in different sets of tissues, arguing against the possibility that a generic "sink" tissue nonspecifically receives any RNAs or proteins present in the circulation.

These findings are most generally of interest to the mouse genetics community. It has long been clear that transgenes carrying Cre under the control of a putatively cell type-specific promoter are often expressed in unexpected tissues [19, 20], and here for example our data confirm prior reports of germline activity of $\mathrm{Nes}^{\mathrm{Cre}}$ and Syn1Cre drivers [21-23]. Our findings also identify many new examples of off target Cre activity for a variety of Cre lines, many of which may have been missed before given the relatively small size of the scientific community focused on the epididymis. In this context our data simply further emphasize the importance of characterizing recombination in conditional knockout studies, as recombination in unanticipated tissues has the potential to confound interpretations of tissue-specific functions of genes of interest. Given the common off target recombination in the epididymis, seminal vesicle and prostate documented here, this is most obviously true for any studies focused on reproductive biology, but as we also find unexpected Cre activity in intestine driven by neuronal Cre drivers we emphasize more general caution when using Cre-Lox genetics in mouse.

The impact of these off target effects will of course depend on the nature of the conditional allele used in a given study. It is worth noting that even in the cases of double transgenics exhibiting robust recombination in the epididymis (Vgat, AdipoQ, etc. - Figures 3-5), we find fairly patchy recombination in the epididymal epithelium. This mosaic recombination behavior has different implications for conditional knockouts, where a large number of epididymis (or seminal vesicle, etc.) cells will escape deletion, vs. gain of function studies where even patchy Cre-dependent induction of gene expression (such as the tdTomato reporter used here) in the target tissue has the potential to exhibit artifactual gain of function.

\section{What is the mechanistic basis for off target activity of neuronal Cre drivers?}

The fact that putatively tissue-specific Cre drivers can often drive recombination in unanticipated tissues has long been known. This is commonly thought to reflect "leaky" expression of a given Cre transgene, potentially owing to the removal of a given promoter from its genomic context (and thus from long-range regulatory elements) in many Cre drivers. In addition, while many promoters chosen as cell-type specific Cre drivers are highly expressed in the cell type of interest, they are often also expressed at lower levels in other cell types throughout the body where the Cre transgene is therefore likely to drive recombination.

That said, our data showing off target Cre activity in the epididymis are unlikely to result from leaky expression of the Cre drivers studied here, for multiple reasons. First of all, we find a wide range of Cre transgenes drive recombination in the epididymis, and most of the neuron-specific genes in question are not detectably expressed in the adult epididymis $[17,18]$. It remains plausible that these genes could be expressed in the epididymis only during early development, before becoming undetectable in the adult epididymis. However, our observation that induction of Cre expression in the brain via AAV-mediated transduction leads to reporter activity in the epididymis epithelium 
(Figure 2, Supplemental Figures S2-3) cannot be explained by leaky transgene expression. Conversely, the possibility that unanticipated tropism of the AAV constructs used here might result in viral transduction of the epididymis would not explain any of the results obtained using double transgenics (Figures 3-5). Together, these findings strongly suggest that Cre activity synthesized in neurons is transported to the epididymis.

The delivery of Cre from neurons to the epididymis could occur via a number of routes, including release of Cre-containing vesicles by peripheral neurons that innervate the cauda epididymis. We consider this to be unlikely for several reasons. First, we show that adipose-specific Cre expression reliably drives recombination in the epididymis (Figure 5), arguing against Cre transfer requiring neuronal termini. Second, induction of Cre expression specifically in one hemisphere of the brain via stereotactic AAV injections was shown to lead to Tomato expression in both the ipsilateral and contralateral epididymis (Figure 2), arguing against short-range neuronal delivery (not to mention the challenges in considering how Cre might move from the CNS to peripheral nerves).

Indeed, our follow-up studies using parabioses and serum transfers support the hypothesis that Cre activity synthesized in a variety of tissues can access the circulation and subsequently be trafficked to the male reproductive tract. Although these efforts to transfer Cre activity from a Cre-expressing animal to a report animal were inefficient, the fact that recombination was ever observed in these studies is best explained by the trafficking hypothesis. We speculate that the circulating Cre activity consists of Cre mRNA, rather than protein, and we anticipate that the RNA is present either in exosomes or in ribonucleoprotein complexes protected from serum nucleases. Indeed, we can document Cre mRNA by PCR in the circulation of multiple Cre lines

(Supplemental Figure S10), and there is abundant precedent for RNA transmission between different tissues. We cannot presently determine whether the transferred Cre activity is contained in exosomes, microvesicles, ribonucleoprotein complexes, or other forms. Again, precedent argues for exosomes as the carriers of inter-tissue molecular information [26], but given the inefficiency of Cre transfer in serum and exosome injections (Figure 6, Supplemental Figure S9) a detailed fractionation effort would require an inordinate number of animals to be used, and must be reserved for future studies.

\section{Potential biological implications}

Our discovery of a pathway by which neurons (and some other tissues) traffic RNAs (or proteins) to the male reproductive tract has exciting possible implications. Most intriguingly, we speculate that environmental conditions experienced by a male could be interpreted by the central nervous system, with salient features of the environment then communicated to the male reproductive tract via this trafficking system. Given the emerging role for the epididymis in remodeling multiple aspects of maturing sperm [2729], an appealing hypothesis would be that receipt of CNS-derived information whether RNAs (mRNAs, lincRNAs, sncRNAs, etc.), proteins, etc. - could then influence the post-testicular maturation of germ cells in the male reproductive tract.

This possibility is particularly intriguing considering the role of systemic RNA trafficking in worms and plants. Most relevant to our study, small RNAs produced in C. 
elegans neurons have been documented to silence genes in the germline and induce transgenerational silencing [3]. Intriguingly, a prior report in mouse suggested that microRNAs expressed in the brain could be delivered to sperm, and subsequently to the next generation [10]. However, with the exception of Syn1 and Nestin (Supplemental Figure S6), we do not find any evidence for neuronal Cre trafficking directly to sperm cells (and in those cases recombination likely occurs either in spermatogonial stem cells or even earlier in development), arguing against the idea that the circulating Cre activity is stably delivered to sperm. Our data instead would place the epididymis as a mediator between neuronal information and programming of the sperm epigenome, with CNSderived information potentially driving altered epididymal physiology and consequent remodeling of sperm small RNAs, proteins, or surface lipids.

An alternative explanation of our findings would be that neuronally-derived molecular information does not have any physiological consequences upon uptake by the epididymis. In such a "trash can" model, the epididymis could simply be unusually susceptible to circulating exosomes or RNPs, with the unique sensitivity of Cre-Lox systems revealing the delivery of Cre molecules at abundances that would likely be meaningless for a typical cellular sncRNA, mRNA, or protein.

We cannot rule out the latter hypothesis at present, but envision two potential approaches to this question in the future. One possibility would be to subject male mice to some stress paradigm or other exposure, then identify changes in transcription or small RNA production in the epididymis. Subsequently, males could be subject to the exposure in question, with circulating serum/exosomes then isolated and injected into an unexposed recipient. In this scenario, if circulating material is responsible for programming epididymal gene expression, the recipient animal would be expected to recapitulate the molecular changes seen in directly-exposed animals. While this approach could be very powerful, our experience with serum/exosome transfers has been that exosome transfer results in very inefficient transfer of Cre activity between animals, with exosomes from nine donor animals required for patchy and lowpenetrance reporter activity (Figure 6, Supplemental Figure S9). This suggests that serum from scores of animals might be required for a single recipient to recapitulate the epididymal changes driven by direct exposures.

An alternative approach would be to identify the molecular basis for epididymal receptivity to neuronal information. For instance, if the relevant carrier of neuronal information is indeed present in exosomes, tetraspanins or other cell surface molecules are presumably responsible for tissue-specific targeting of exosomes to the epididymis. Identification of the relevant exosomal receptor could then be used in knockout studies to prevent receipt of neuronal shipments, and changes in epididymal responses to various organismal stressors could then be used to identify potential effects of neuronal RNA/protein shipments on epididymal physiology.

\section{Conclusions}

Here, we have demonstrated that Cre activity synthesized in neurons and in adipose tissue consistently directs recombination in the epididymis - with some lines also having other off target tissues. These findings add to the literature on the dangers of Cre-Lox genetics in conditional knockout studies, identifying additional off target tissues for a number of Cre drivers. In addition, our data suggest that this poor tissue specificity 
could be caused in part by mobilization of Cre activity through the circulation, implying a surprising mechanism behind some of these off target effects. Finally, the possibility that this pathway plays a role in sensing of environmental conditions in modulation of the sperm epigenome represents an exciting hypothesis for future studies in paternal effect epigenetics.

\section{ACKNOWLEDGEMENTS}

We thank Guanping Gao for the generous gift of AAV9-CMV-Cre, Andrew Recknagel for tissue and images, and Dr Rebecca Williams and grant S10 OD023466 - A Light Sheet Microscope for the Cornell BRC Imaging Facility - for lightsheet microscopy help. We thank I. Bach, R. Davis, and D. Weaver for generous gift of several Cre driver lines, and $\mathrm{D}$. Weaver and $\mathrm{P}$ Visconti for critical reading of the manuscript. This work was funded by the Templeton Foundation (Grant 61350), and NICHD (R01HD080224) to OR and NIH (DP1 AG063419) to AJW.

\section{CONFLICT OF INTEREST}

AJW is a scientific advisor for Kate Therapeutics and Frequency Therapeutics and a cofounder, advisor, and holder of private equity in Elevian, Inc., which also provides sponsored research to the Wagers Lab. AJW is an inventor on various patents that include the use of AAVs for research and therapeutic applications. The remaining authors declare no competing interests.

\section{MATERIALS AND METHODS}

\section{Mouse husbandry}

All animal use was approved by the Institutional Animal Care and Use Committees of UMass Chan Medical School and Harvard Faculty of Arts and Sciences, under protocol PROTO202100029 to Dr. Oliver Rando and protocol 29014 awarded to Dr. Amy Wagers.

The transgenic mice utilized in this study were maintained by filial matting, giving preference toward homozygous carriers whenever possible. To avoid introduction of genetic confounders the breeding colonies were maintained for up to 3 generations, after which animals were either backcrossed to their background strain or the colony was culled, and new breeding pairs acquired.

Below is a list with the official names for all the mice strains and the genotype abbreviations used throughout this text, as well as the strain number listed at the Jackson Laboratory.

\begin{tabular}{|l|l|l|l|l|}
\hline Transgenic allele & Expression of & Promoter & JAX Catalog \# & $\begin{array}{l}\text { Genotype } \\
\text { abbreviation }\end{array}$ \\
\hline $\begin{array}{l}\text { B6.Cg-Gt(ROSA)26Sortm14(CAG- } \\
\text { tdTomato)Hze/J }\end{array}$ & $\begin{array}{l}\text { TdTomato } \\
\text { upon Cre }\end{array}$ & Chicken Actin Gene & 007914 & Ai14D \\
\hline Slc32a1tm2(cre)Lowl/JW & Cre & $\begin{array}{l}\text { Vgat - GABA vesicular } \\
\text { transporter }\end{array}$ & 028892 & Vgat \\
\hline
\end{tabular}




\begin{tabular}{|c|c|c|c|c|}
\hline B6.Cg-Tg(Nes-cre) $1 \mathrm{Kln} / \mathrm{J}$ & Cre & Nestin & 3771 & Nes ${ }^{\mathrm{Cre}}$ \\
\hline $\begin{array}{l}\text { B6N.Cg-Speer6-ps1Tg(Alb- } \\
\text { cre)21Mgn/JR }\end{array}$ & Cre & Albumin & 003574 & $A / b^{C r e}$ \\
\hline B6.FVB-Tg(Adipoq-cre)1Evdr/J & Cre & Adiponectin & 028020 & AdipoQ $Q^{\text {Cre }}$ \\
\hline $\begin{array}{l}\text { B6;129-Tg(Drd1a- } \\
\text { cre)120Mxu/MmjaxT }\end{array}$ & Cre & $\begin{array}{l}\text { Drd1a-dopamine } \\
\text { receptor G-protein }\end{array}$ & 037156 & $\operatorname{Drd1} 1^{\text {Cre }}$ \\
\hline B6N.Cg-Gad2tm2(cre)Zjh/JT & Cre & $\begin{array}{l}\text { Gad2 - glutamic acid } \\
\text { decarboxylase } 2\end{array}$ & 010802 & $\operatorname{Gad2} 2^{\text {cre }}$ \\
\hline B6.Cg-Tg(Fev-cre)1Esd/JT & Cre & $\begin{array}{l}\text { Fev - ETS oncogene } \\
\text { family }\end{array}$ & 012712 & Fev ${ }^{C r e}$ \\
\hline B6.Cg-Tg(Syn1-cre)671Jxm/J & Cre & Synapsin1 & 003966 & Syn1 $1^{\text {Cre }}$ \\
\hline STOCK Defb41tm1(icre)Psip/R & iCre & $\begin{array}{l}\text { Defb41 - Defensin } \\
\text { beta } 41\end{array}$ & $\begin{array}{l}\text { Gift from P. } \\
\text { Sipila }\end{array}$ & Defb41 $1^{i c r e}$ \\
\hline B6.C-Tg(CMV-cre)1Cgn/J & Cre & CMV & 006054 & CMV Cre \\
\hline
\end{tabular}

All genotypes were determined using Transnetyx genotyping services.

\section{AAV constructs}

An initial set of three animals were injected with rAAV2-retro-Eif1a-Cre. Subsequent experiments used an AAV9-based construct carrying pCMV-CRE: AAV9.CB-PI-Cre $5.03 \times 10^{12} \mathrm{GC} / \mathrm{mL} ; \mathrm{VCAV}-04562$. Virus was a gift from the Gao lab.

\section{Stereotaxic AAV injections}

Stereotaxic injections were performed to deliver adeno associated virus (AAV) constructs into different regions of the central nervous system of adult Ai14D males. Adult male mice ( 8 to 12 weeks old) were anaesthetized with a mixture of $100 \mathrm{mg} / \mathrm{kg}$ ketamine and $10 \mathrm{mg} / \mathrm{kg}$ xylazine, administered via intraperitoneal (IP) injection. Prior to surgery, the top of the skull was shaved and disinfected. Surgeries were performed using aseptic technique with the aid of a stereotaxic frame (Stoelting Co.). Mice were placed into the stereotaxic frame and the skull was exposed by making a small incision with a scalpel blade. Using bregma and lambda as landmarks, the skull was then levelled along the coronal and sagittal planes. Two small drill holes were made in the skull that allowed injections to target the somatosensory cortex, caudate putamen, and nucleus accumbens. Microinjections were made using a Hamilton $10 \mu \mathrm{l}$ neurosyringe (1701RN; Hamilton) and a microsyringe pump (Stoelting Co). $0.5 \mu \mathrm{l}$ of virus was delivered through the syringe and at a constant flow rate of $30 \mathrm{nl} / \mathrm{min}$. After injection, the needle was left unmoved for 10 min before being slowly retracted. The incision was then closed and held together with glue.

\section{Tissue collection and preparation}

\section{Transcardiac perfusion}

To prepare tissues for imaging, animals were subject to transcardiac perfusion under deep anesthesia. Once deep plane of anesthesia, as assessed by toe pinch, was established, the thoracic cavity was open to expose the heart and using an insulin syringe with 31 gauge needle heparin was directly infused into the left ventricle. A 
butterfly needle hooked to a peristaltic pump was secured in the left ventricle and the right atrium nicked to allow perfusion with $20 \mathrm{~mL}$ PBS, followed by $25 \mathrm{~mL}$ of freshly prepared $4 \%$ paraformaldehyde. Harvested organs were incubated in $4 \%$ PFA overnight, then washed with PBS and either transferred to $30 \%$ sucrose for cryoprotection (followed by OCT embedding for histology) or subjected to tissue clearing for light sheet imaging.

For tissue clearing, passive de-lipidation performed by incubating the organ with $4 \%$ SDS/PBS at RT in an orbital shaker for three weeks [30]. The 4\% SDS/PBS solution was changed every other day. At the end of the third week, the organ was rinsed with PBS for three times of least $4 \mathrm{hr}$ and finally placed on a refractive index matching solution (0.17M iodixanol; 0.4M diatrizoic acid, $1 \mathrm{M}$ n-methyl-d-glucamine, $0.01 \%$ sodium azide) $24 \mathrm{hr}$ prior to imaging. Light-sheet images were acquired using LaVisionBioTec LightSheet Microscopy Ultramicroscope II located at the Cornell Imaging facility, IthacaNY. Images were processed using Arivis 4D software.

\section{Parabioses}

Male mice of similar age from two different strains - one "donor" animal expressing Cre, and the Ai14D Tom ${ }^{f / x} / f x$ "recipient" - were surgically connected to allow the sharing of their circulatory systems. Surgical preparations were performed on the shaved skin, where incision was made along the opposing flanks of each mouse to be joined. A skin flap was made without damaging the underlying peritoneal lining, and the mice placed side-by-side in a prone position. To better secure the connection, the animals' joints (elbows and knees) were held together using suture. The dorsal and ventral skin flaps from the different mice were then pinched together and sutured. Parabiotic pairs were maintained for eight weeks after surgery and therefore expected to have maintained cross-circulation for at least two weeks [31].

\section{Serum and exosome injections}

\section{Serum Collection}

Blood was collected from the right ventricle of the heart of a deeply anesthetized animals using a $3 \mathrm{ml}$ syringe 27 -gauge needle. If serum was to be used for tangential flow filtration (TFF), ultracentrifugation or RNA extraction, a more thorough exsanguination was achieved by injection of $1.5 \mathrm{ml}$ of saline solution using a 31 -gauge needle into left ventricle, thus pushing more blood to the right ventricle for collection. The blood was transferred to the yellow cap serum collection tubes and placed on ice until the end of the collection. Once all animals were processed, serum was obtained after incubating samples for $20 \mathrm{~min}$ at room temperature and performing a $9000 \mathrm{~g}$ spin for $10 \mathrm{~min}$ followed by $12000 \mathrm{~g}$ for 5 minutes. Serum was transferred to a clean $1.5 \mathrm{ml}$ Eppendorf tube further centrifuged at $4 \mathrm{C}$ for $10 \mathrm{~min}$ at $16000 \mathrm{~g}$, and serum transferred to a new tube and either used for intravenous injections or stored at $-80 \mathrm{C}$ for later processing.

\section{Tangential flow filtration (TFF) exosome enrichment}

We use the manual TFF-easy from Galen molecular laboratory supplies. The device is a filter cartridge with polysulfone hollow fibers with pores of $5 \mathrm{~nm}$ diameter along its length. It allows the concentration of extra cellular vesicles by the removal of small proteins, 
molecules, and solutes from serum. Water and small molecules up to $5 \mathrm{~nm}$ pass through the pores and this fluid is discarded as flowthrough thus allowing the concentration of the serum EVs.

\section{https://galenmolecular.com/exosome-products/exosome-isolation/tff-easy/}

\section{Injection schedules}

Serum and exosome injections were performed using serum/exosomes isolated from a variety of Cre lines, with at least three tail vein injections per recipient spread across five days (eg every other day). Across all injection schemes, epididymal recombination was observed only in a subset of recipients (Figure 6 and Supplemental Figure S9). We therefore continuously modified the injection scheme used - hoping to identify reproducible conditions for transfer of Cre activity - with various recipients receiving serum only, exosomes only, or various mixtures of the two. However, we have been unable to identify experimental variables that distinguish successful (as defined by reporter expression in the recipient animal) from unsuccessful transfers.

\section{Histology, tissue clearing and light sheet microscopy Histology}

Male mice from the same strain and age as previously described were perfused with $4 \%$ paraformaldehyde (PFA)/PBS, and epididymi explanted and further incubated in fixative at $4 \mathrm{C}$ overnight $(\mathrm{ON})$. After washing the excess of PFA with PBS, the sample was incubated at $4 \mathrm{C}$ with a $30 \%$ sucrose, $0.002 \%$ sodium azide in PBS solution for 32 hours, or until tissues sank to the bottom of the container. Prior to mounting in OCT, sucrose was replaced with a one-to-one solution of $30 \%$ sucrose to OCT and samples kept at $4 \mathrm{C}$ with agitation. Samples were than mounted using OCT. Cryo-sections were done at 5-10 $\mu \mathrm{m}$ thickness by the UMASS morphology core. Slides were store at $-80 \mathrm{C}$ until further processing.

\section{Fluorescence}

Frozen slides were incubated at a $37 \mathrm{C}$ warm plate for at least 10 minutes to ensure proper attachment of the section to the slide, then washed three $X$ five minutes in PBS $0.02 \%$ tween 20 (PBS-T) to remove OCT. For the nuclear visualization, slides were immersed in a PBS solution containing $3 \mu \mathrm{M}$ DAPI (4',6-diamidino-2-phenylindole in PBS) for $5 \mathrm{~min}$. Afterwards slides were rinsed and mounted with ProLong Antifade gold (Thermofisher) and imaged on an Automated Zeiss Axioobserver workstation with DIC, LED fluorescence, autofocus, and deconvolution image processing.

\section{FLOW}

Single cell suspension of the epididymis were performed as previously described [18]. Briefly, epididymis samples were separated into caput corpus cauda and vas and enzymatically dissociated. Cells were resuspended in HSS and transferred to a $1.5 \mathrm{ml}$ Eppendorf tube for staining with viability dye and nuclear stain. Afterwards the cell suspention was once more filtered while being transferred to a $5 \mathrm{ml}$ polypropylene FACS tube, place in ice and protected from light until flow cytometry data acquisition. Data acquisition was performed in Cytec or LSRII with PMT voltages adjusted for each 
experimental day using single stained cells from homozygous Ai14D mice as well as from ubiquitously Cre-expressing Ai14D mice. Fluoresce Minus One (FMO) was also made. No compensation was necessary.

\section{PCR assay for recombination}

Recombination PCR was done using 2X MyTaq Red Mix (Meridian Biosciences, cat. BIO-25044) per manufacturer's instructions. Primers were diluted to final concentrations of $0.33 \mu \mathrm{M}$ for Rec7 and $0.67 \mu \mathrm{M}$ for Rec11 and SeqRec. 200ng DNA was used per reaction. PCR products were run on $2 \%$ agarose gel.

\section{High throughput RNA/DNA extraction.}

Slightly modified from the described steps on the Qiagen RNA\&DNA extraction protocol. Namely, mandatory use of the antifoam reagent and adoption of a fixed $1 \mathrm{ml}$ volume of lysis buffer to $3 \mathrm{~mm}^{3}$ of tissue. The best lysis was obtained using bead beater with the pre-programmed settings for kidney mouse tissue repeated 3 times and starting every cycle on frozen tissue/lysis buffer.

\section{FIGURE LEGENDS}

Figure 1. Analysis of Cre-dependent reporter gene expression in the murine epididymis

A) Schematic of the Cre-dependent reporter present in the Ai14D strain. Reporter cassette, inserted at the safe harbor Rosa26 locus, carries a strong pCAG promoter followed by a LoxP-flanked STOP cassette with three repeated in frame sequences. In the absence of Cre activity, transcription through this construct leads to transcriptional termination and no reporter activity. Following Cre-dependent excision of the LoxP cassette, the pCAG promoter is juxtaposed adjacent to a sequence encoding a tandem Tomato (tdTomato) fluorescent protein, resulting in robust expression of Tomato in tissues expressing Cre.

B) Light sheet imaging of the mouse epididymis in the negative control Ai14D background. Following animal sacrifice, tissues were cleared for imaging according to a modified CLARITY protocol (Methods). The entire epididymis, along with a large section of the vas deferens, was then imaged using the LaVision Biotec light sheet microscope. Regions corresponding to the proximal (caput), middle (corpus), and distal (cauda) epididymis, and the vas deferens, are indicated on the image of the entire tissue sample. Insets show representative 2D slices of the indicated anatomical regions. C) Positive control showing robust Tomato expression driven by the caput-specific Defb41 Cre driver [16]. Panels show whole tissue image and representative sections as described in panel (B), along with representative sections of the kidney and liver, as indicated. See also Supplemental Figure S1.

Figure 2. AAV-mediated Cre expression in the brain drives reporter expression in the epididymis

A) Experimental schematic showing stereotactically-guided injection of AAV into the brain of Ai14D reporter animals to drive localized Cre-Lox recombination in the brain. 15 
or 25 days post injection (depending on the experiment), mice were sacrificed and subject to tissue clearing and analysis by lightsheet microscopy.

B) Tomato reporter activity in the brain of typical AAV-injected animal. Panel shows maximum intensity projection for the entire mouse brain, viewed from above.

C) Images of the epididymis, as in Figures 1B-C. Right panels show two distinct 2D slices of the cauda epididymis, highlighting the Tomato-positive rim surrounding the Tomato-negative lumen. See also Supplemental Figures S2-S3.

Figure 3. Cre expression in GABAergic neurons drives reporter expression in the epididymis

A) Schematic showing the cross that generated the double transgenic analyzed here, generated via crossing the Ai14D reporter with a strain bearing the Vgat Cre transgene (Methods).

B) Lightsheet images of the indicated tissues in Ai14D; Vgat Cre double transgenic animals.

C) Lightsheet images of the epididymis in Ai14D; VgatCre double transgenic animals.

D) Two representative histology sections from Ai14D; VgatCre cauda epididymis and vas deferens, counterstained with DAPI, clearly showing Tomato-positive principal cells in the epithelium. See also Supplemental Figures S4-5.

E) Recombination at the reporter locus in Ai14D; Vgat ${ }^{\text {Cre }}$ tissues. Top: Schematic shows location of primers used on the PCR. Bottom: Each gel shows 6 tissues from a negative control Ai14D animal (no exposure to Cre), along with 6 tissues from the Ai14D; VgatCre double transgenic. POS: positive control tissue (liver from an Ai14D; Alb ${ }^{\text {Cre }}$ transgenic). Red arrow indicates the band arising from the recombined locus, with clear recombination observed in the brain, as well as all epididymal samples, in Ai14D; VgatCre animals.

Figure 4. Multiple neuronal Cre drivers result in reporter activity in the epididymis A) Schematic showing the cross that generated the double transgenics analyzed here, as in Figure 3A.

B) Representative lightsheet images of the epididymis from the indicated double transgenic animals. (See also Supplemental Figures S5-8).

Figure 5. Reporter activity in Adipo $Q^{\text {Cre }}$ animals

A) Schematic, as in Figures 3A, 4A.

B) Histology from Ai14D; AdipoQ ${ }^{\text {Cre }}$ animals, showing Tomato expression scattered throughout the epididymis epithelium.

C) PCR analysis of indicated tissues of Ai14D; AdipoQCre animals, revealing robust recombination in the epididymis. POS: positive control tissue. Red arrow indicates the band arising from the recombined locus.

Figure 6. Cre activity present in the circulation can be transferred from a Cre driver to a reporter animal

A) Experimental schematic. Parabiosis surgery was used to link the circulatory systems of an Ai14D reporter animal and that of a $\mathrm{Vgat}^{\mathrm{Cre}}$ or $\mathrm{Nes}^{\mathrm{Cre}}$ animal. After 8-9 weeks 
together, animals were sacrificed and tissues from both animals were obtained for analysis.

B) Histology from the indicated sides (highlighted in red) of two Ai14D::Cre parabiotic pairs. We observe Tomato expression specifically on the reporter side, but not the Cre side, of Ai14D::Vgat Cre animals.

C) Genotyping PCR from the Ai14D side, and the Cre side, of the indicated parabiotic pairs, with faint recombination detected in the cauda epididymis of Ai14D:: Vgat Cre animals.

D) Schematic showing serum/exosome transfer experiments. Note that these experiments were carried out with a wide range of injection schedules (Methods), using both whole serum transfers as well as TFF-enriched exosome transfers for recipient animals.

E) Lightsheet images of Ai14D recipient following transfer of serum and exosomes from $A / b^{\text {Cre }}$ donors (left panels), or from Vgat ${ }^{\text {Cre }}$ donors (right panels).

F) Genotyping PCR from Ai14D animals receiving serum or TFFs from the indicated Cre donor lines. Red arrow indicates the band diagnostic of a recombined locus.

\section{SUPPLEMENTAL MATERIALS}

Supplemental Figures S1-S10

Supplemental Table S1

\section{SUPPLEMENTAL FIGURE LEGENDS}

Supplemental Figure S1. Histology of Ai14D; Defb41 ${ }^{\text {Cre }}$ double transgenic DAPI-stained histological section of the caput and corpus epididymis from an Ai14D; Defb 41Cre animal, showing expected tdTomato-expression specifically in the principal cells of caput epididymis. Top panel shows the entire caput and corpus, bottom panel shows a zoom-in from the distal caput.

\section{Supplemental Figure S2. Additional examples of tomato-positive epididymis samples from reporter animals subject to AAV-mediated Cre expression in the CNS}
A) AAV injection scheme. As in Figure 2, but with rAAV2-retro-Eif1a-Cre injections into the prefrontal cortex.
B) Lightsheet images of the entire epididymis from two animals injected with rAAV2- retro-Eif1a-Cre in the prefrontal cortex.
C) Individual 2D cross sections from the cauda epididymis from the samples shown in panel (B).

\section{Supplemental Figure S3. Fluorescence microscopy images of epididymis from reporter animals subject to AAV-mediated Cre expression in the CNS}
A) Microscopy images of the cauda epididymis from animals mock-injected with saline, as indicated in the accompanying schematic. 
B) As in panel (A), but for animals injected with AAV9-CMV-Cre in the lateral somatosensory cortex, caudate putamen, and nucleus accumbens (see also Figure 2).

\section{Supplemental Figure S4. Flow cytometry analysis of Tomato-positive cells in the epididymis}

Bar charts show \% Tomato-positive cells obtained from the indicated tissue samples, in negative control Ai14D animals (grey bars) or two double transgenic animals (red bars), as indicated. Both $\mathrm{Vgat}^{\mathrm{Cre}}$ and $\mathrm{Nes}^{\mathrm{Cre}}$ drove an increase in Tomato-positive cell numbers in the epididymis, consistent with our lightsheet, histology, and genotyping studies.

\section{Supplemental Figure S5. Minimal off-target recombination driven by $A / b^{C r e}$.}

A) Experimental schematic of mating strategy.

B) Histology of the cauda and corpus epididymis from Ai14D; Alb ${ }^{\text {Cre }}$ double transgenic animal. Across the entire section we find two isolated Tomato-positive cells in this animal.

C) PCR assay for recombination in the indicated tissues obtained from Ai14D; AlbCre double transgenic animals. A light recombination band is seen in the cauda epididymis, consistent with low level reporter activity in panel (B), while we find no evidence for any other off-target recombination.

Supplemental Figure S6. Tomato expression in epididymis samples from Ai14D animals carrying pan-neuronal Cre drivers.

A) Ai14D; Syn1Cre double transgenic sample. Abundant Tomato signal here is clearly localized to the lumen of the epididymal tubule, consistent with Tomato expression in the male reproductive tract and potentially in the sperm, resulting from germline recombination in this animal.

B) Ai14D; Nes ${ }^{\text {Cre }}$ double transgenic sample. Here, histology images shown widespread principal cell Tomato expression, similar to that observed in double transgenic Ai14D; Vgat ${ }^{\text {Cre }}$ animals (Figure 3D), but with positive cells being found throughout all regions of the epididymis (in contrast to the cauda and vas deferens-enriched recombination in the Vgat Cre animals).

\section{Supplemental Figure S7. Examples of off-target Cre activity beyond the epididymis.}

Lightsheet images of the indicated tissues showing Tomato expression in the various Ai14D; Cre double transgenic lines, as indicated.

Supplemental Figure S8. Off target reporter activity in accessory glands. Lightsheet images of the accessory glands are shown to the left, along with three representative sections in the right panels, for the two indicated Cre driver lines.

\section{Supplemental Figure S9. Inconsistent Cre transfer via parabiosis or serum} transfer experiments.

A) Parabiosis schematic. 
B) Histology images of the cauda epididymis from the Ai14D recipient animal after eight weeks in a parabiosis with a VgatCre donor. Zoom in images show Tomato-positive epithelial cells in cauda epididymis.

C) Genotyping PCR for the indicated samples. The first three samples were gathered from the indicated double transgenic. For the two parabiotic animal pairs here, samples from the Ai14D recipient and the Cre donor were each assayed.

D) Serum/exosome transfer schematic.

E) Lightsheet images from eight serum/exosome recipients, with the Cre driver used as the serum/exosome donor indicated for each sample. Tomato-positive and -negative samples are organized separately. Note that one recipient received exosomes from both $\mathrm{Gad} 2 \mathrm{Cre}$ donor and $\mathrm{Drd} 1 \mathrm{1}$ re donors on different injection days.

F) Genotyping PCR for recombination in the indicated serum/exosome recipients. Most recipients were negative for recombination, with strong recombination in one recipient of Adipo $\mathrm{Q}^{\mathrm{Cre}}$ exosomes, and very weak recombination observed in one recipient of $\mathrm{Nes}^{\mathrm{Cre}}$ exosomes.

\section{Supplemental Figure S10. Cre-encoding mRNA present in the circulation of various Cre driver animals. \\ RT-PCR using Cre-specific primers. Samples include water controls, and RNA from either serum or exosomes (indicated in black and red, respectively) isolated from the indicated Cre lines.}

\section{SUPPLEMENTAL TABLE LEGENDS}

\section{Supplemental Table S1. Oligonucleotides used in this study}

\begin{tabular}{|c|c|c|c|c|}
\hline \multicolumn{2}{|c|}{ Primer Set } & Sequence & $\begin{array}{l}\text { Full-length } \\
\text { (bp) }\end{array}$ & $\begin{array}{l}\text { Recombined } \\
\text { (bp) }\end{array}$ \\
\hline \multirow[t]{2}{*}{$\operatorname{Rec} 7$} & Forward & $\begin{array}{l}\text { GCA ACG TGC TGG TTA TTG } \\
\text { TG }\end{array}$ & \multirow[t]{2}{*}{1118} & \multirow[t]{2}{*}{248} \\
\hline & Reverse & $\begin{array}{l}\text { CTC ACC ATG GTG GCG GGA } \\
\text { TC }\end{array}$ & & \\
\hline \multirow[t]{2}{*}{ Rec11 } & Forward & $\begin{array}{l}\text { GGT TCG GCT TCT GGC GTG } \\
\text { TGA CC }\end{array}$ & \multirow[t]{2}{*}{1202} & \multirow[t]{2}{*}{332} \\
\hline & Reverse & $\begin{array}{l}\text { CTC ACC ATG GTG GCG GGA } \\
\text { TC }\end{array}$ & & \\
\hline \multirow[t]{2}{*}{ SeqRec } & Forward & $\begin{array}{l}\text { GCA ACG TGC TGG TTA TTG } \\
\text { TG }\end{array}$ & \multirow[t]{2}{*}{1711} & \multirow[t]{2}{*}{807} \\
\hline & Reverse & $\begin{array}{l}\text { CCA GCT TGG TGT CCA CGT } \\
\text { AGT A }\end{array}$ & & \\
\hline
\end{tabular}

\section{REFERENCES}

1. Fire A, Xu S, Montgomery MK, Kostas SA, Driver SE, Mello CC. Potent and specific genetic interference by double-stranded RNA in Caenorhabditis elegans. Nature.

1998;391(6669):806-11. PubMed PMID: 9486653. 
2. Timmons L, Fire A. Specific interference by ingested dsRNA. Nature. 1998;395(6705):854. doi: 10.1038/27579. PubMed PMID: 9804418.

3. Devanapally S, Ravikumar S, Jose AM. Double-stranded RNA made in C. elegans neurons can enter the germline and cause transgenerational gene silencing. Proceedings of the National Academy of Sciences of the United States of America. 2015;112(7):2133-8. doi: 10.1073/pnas.1423333112. PubMed PMID: 25646479; PubMed Central PMCID: PMCPMC4343102.

4. Palauqui JC, Elmayan T, Pollien JM, Vaucheret H. Systemic acquired silencing: transgene-specific post-transcriptional silencing is transmitted by grafting from silenced stocks to non-silenced scions. The EMBO journal. 1997;16(15):4738-45. doi: 10.1093/emboj/16.15.4738. PubMed PMID: 9303318; PubMed Central PMCID: PMCPMC1170100.

5. Voinnet O, Vain P, Angell S, Baulcombe DC. Systemic spread of sequence-specific transgene RNA degradation in plants is initiated by localized introduction of ectopic promoterless DNA. Cell. 1998;95(2):177-87. doi: 10.1016/s0092-8674(00)81749-3. PubMed PMID: 9790525.

6. van Niel G, D'Angelo G, Raposo G. Shedding light on the cell biology of extracellular vesicles. Nature reviews Molecular cell biology. 2018;19(4):213-28. doi: 10.1038/nrm.2017.125. PubMed PMID: 29339798.

7. Thomou T, Mori MA, Dreyfuss JM, Konishi M, Sakaguchi M, Wolfrum C, et al. Adipose-derived circulating miRNAs regulate gene expression in other tissues. Nature. 2017;542(7642):450-5. doi: 10.1038/nature21365. PubMed PMID: 28199304; PubMed Central PMCID: PMCPMC5330251.

8. Poggio M, Hu T, Pai CC, Chu B, Belair CD, Chang A, et al. Suppression of Exosomal PD-L1 Induces Systemic Anti-tumor Immunity and Memory. Cell. 2019;177(2):414-27 e13. doi: 10.1016/j.cell.2019.02.016. PubMed PMID: 30951669; PubMed Central PMCID: PMCPMC6499401.

9. Cossetti C, Lugini L, Astrologo L, Saggio I, Fais S, Spadafora C. Soma-to-germline transmission of RNA in mice xenografted with human tumour cells: possible transport by exosomes. PloS one. 2014;9(7):e101629. doi: 10.1371/journal.pone.0101629. PubMed PMID: 24992257; PubMed Central PMCID: PMCPMC4081593.

10. O'Brien EA, Ensbey KS, Day BW, Baldock PA, Barry G. Direct evidence for transport of RNA from the mouse brain to the germline and offspring. BMC Biol. 2020;18(1):45. doi: 10.1186/s12915-020-00780-w. PubMed PMID: 32354330; PubMed Central PMCID: PMCPMC7191717.

11. Conine CC, Rando OJ. Soma-to-germline RNA communication. Nature reviews Genetics. 2021. doi: 10.1038/s41576-021-00412-1. PubMed PMID: 34545247.

12. Madisen L, Zwingman TA, Sunkin SM, Oh SW, Zariwala HA, Gu H, et al. A robust and high-throughput Cre reporting and characterization system for the whole mouse brain. Nature neuroscience. 2010;13(1):133-40. doi: 10.1038/nn.2467. PubMed PMID: 20023653; PubMed Central PMCID: PMCPMC2840225.

13. Kauffman KJ, Oberli MA, Dorkin JR, Hurtado JE, Kaczmarek JC, Bhadani S, et al. Rapid, Single-Cell Analysis and Discovery of Vectored mRNA Transfection In Vivo with a loxP-Flanked tdTomato Reporter Mouse. Mol Ther Nucleic Acids. 2018;10:55-63. doi: 10.1016/j.omtn.2017.11.005. PubMed PMID: 29499956; PubMed Central PMCID: PMCPMC5734870. 
14. Chung K, Deisseroth K. CLARITY for mapping the nervous system. Nature methods. 2013;10(6):508-13. doi: 10.1038/nmeth.2481. PubMed PMID: 23722210.

15. Cronan MR, Rosenberg AF, Oehlers SH, Saelens JW, Sisk DM, Jurcic Smith KL, et al. CLARITY and PACT-based imaging of adult zebrafish and mouse for whole-animal analysis of infections. Dis Model Mech. 2015;8(12):1643-50. doi: 10.1242/dmm.021394. PubMed PMID: 26449262 ; PubMed Central PMCID: PMCPMC4728314.

16. Bjorkgren I, Saastamoinen L, Krutskikh A, Huhtaniemi I, Poutanen M, Sipila P. Dicer1 ablation in the mouse epididymis causes dedifferentiation of the epithelium and imbalance in sex steroid signaling. PloS one. 2012;7(6):e38457. doi: 10.1371/journal.pone.0038457. PubMed PMID: 22701646; PubMed Central PMCID: PMCPMC3368854.

17. Johnston DS, Jelinsky SA, Bang HJ, DiCandeloro P, Wilson E, Kopf GS, et al. The mouse epididymal transcriptome: transcriptional profiling of segmental gene expression in the epididymis. Biology of reproduction. 2005;73(3):404-13. PubMed PMID: 15878890.

18. Rinaldi VD, Donnard E, Gellatly KJ, Rasmussen M, Kucukural A, Yukselen O, et al. An atlas of cell types in the mammalian epididymis and vas deferens. BioRxiv. 2020. doi: https://doi.org/10.1101/2020.01.24.918979

19. Song AJ, Palmiter RD. Detecting and Avoiding Problems When Using the Cre-lox System. Trends Genet. 2018;34(5):333-40. doi: 10.1016/j.tig.2017.12.008. PubMed PMID: 29336844; PubMed Central PMCID: PMCPMC5910175.

20. Stifter SA, Greter M. STOP floxing around: Specificity and leakiness of inducible Cre/loxP systems. Eur J Immunol. 2020;50(3):338-41. doi: 10.1002/eji.202048546. PubMed PMID: 32125704.

21. Luo L, Ambrozkiewicz MC, Benseler F, Chen C, Dumontier E, Falkner S, et al. Optimizing Nervous System-Specific Gene Targeting with Cre Driver Lines: Prevalence of Germline Recombination and Influencing Factors. Neuron. 2020;106(1):37-65 e5. doi: 10.1016/j.neuron.2020.01.008. PubMed PMID: 32027825; PubMed Central PMCID: PMCPMC7377387.

22. Betz UA, Vosshenrich CA, Rajewsky K, Muller W. Bypass of lethality with mosaic mice generated by Cre-loxP-mediated recombination. Current biology : CB. 1996;6(10):1307-16. doi: 10.1016/s0960-9822(02)70717-3. PubMed PMID: 8939573.

23. McLeod VM, Cuic B, Chiam MDF, Lau CL, Turner BJ. Exploring germline recombination in Nestin-Cre transgenic mice using floxed androgen receptor. Genesis. 2020;58(10-11):e23390. doi: 10.1002/dvg.23390. PubMed PMID: 32744751.

24. Gibney BC, Chamoto K, Lee GS, Simpson DC, Miele LF, Tsuda A, et al. Crosscirculation and cell distribution kinetics in parabiotic mice. J Cell Physiol. 2012;227(2):821-8. doi: 10.1002/jcp.22796. PubMed PMID: 21503883; PubMed Central PMCID: PMCPMC3160515.

25. Heinemann ML, Ilmer M, Silva LP, Hawke DH, Recio A, Vorontsova MA, et al. Benchtop isolation and characterization of functional exosomes by sequential filtration. $\mathrm{J}$ Chromatogr A. 2014;1371:125-35. doi: 10.1016/j.chroma.2014.10.026. PubMed PMID: 25458527.

26. Valadi H, Ekstrom K, Bossios A, Sjostrand M, Lee JJ, Lotvall JO. Exosome-mediated transfer of mRNAs and microRNAs is a novel mechanism of genetic exchange between cells. Nature cell biology. 2007;9(6):654-9. PubMed PMID: 17486113. 
27. Gervasi MG, Visconti PE. Molecular changes and signaling events occurring in spermatozoa during epididymal maturation. Andrology. 2017;5(2):204-18. doi:

10.1111/andr.12320. PubMed PMID: 28297559; PubMed Central PMCID: PMCPMC5354101.

28. Tamessar CT, Trigg NA, Nixon B, Skerrett-Byrne DA, Sharkey DJ, Robertson SA, et al. Roles of male reproductive tract extracellular vesicles in reproduction. Am J Reprod Immunol. 2021;85(2):e13338. doi: 10.1111/aji.13338. PubMed PMID: 32885533.

29. Zhou W, De Iuliis GN, Dun MD, Nixon B. Characteristics of the Epididymal Luminal Environment Responsible for Sperm Maturation and Storage. Front Endocrinol (Lausanne). 2018;9:59. doi: 10.3389/fendo.2018.00059. PubMed PMID: 29541061; PubMed Central PMCID: PMCPMC5835514.

30. Rinaldi VD, Bloom JC, Schimenti JC. Whole Mount Immunofluorescence and Follicle Quantification of Cultured Mouse Ovaries. J Vis Exp. 2018;(135). doi: 10.3791/57593. PubMed PMID: 29782020; PubMed Central PMCID: PMCPMC6101098.

31. Wright DE, Wagers AJ, Gulati AP, Johnson FL, Weissman IL. Physiological migration of hematopoietic stem and progenitor cells. Science (New York, NY. 2001;294(5548):1933-6. doi: 10.1126/science.1064081. PubMed PMID: 11729320. 
A
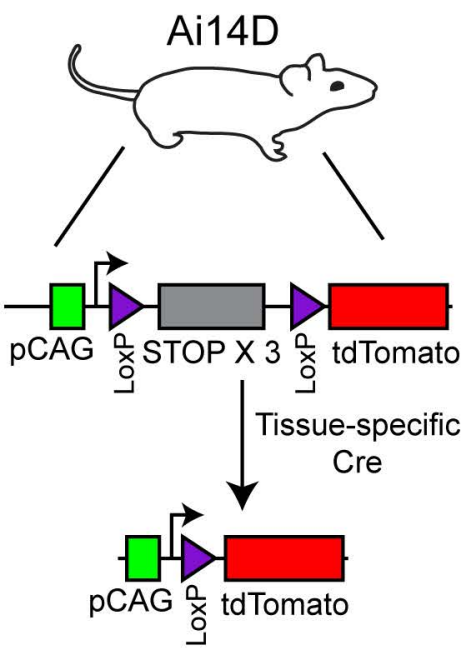

B

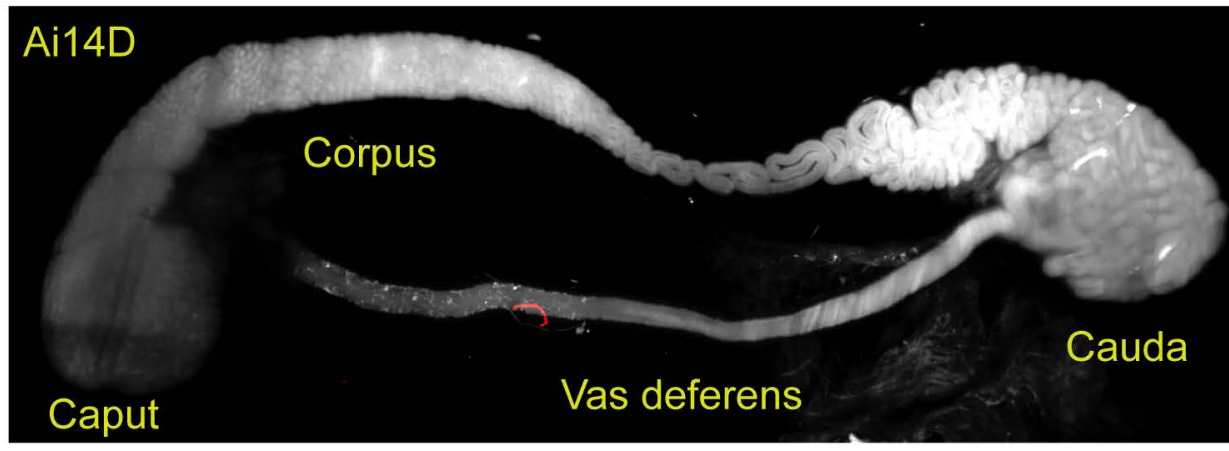

Caput

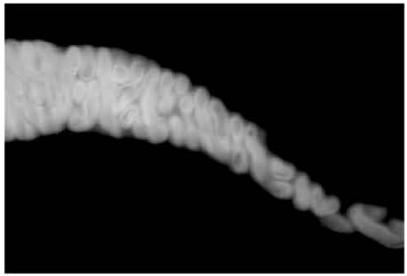

Corpus

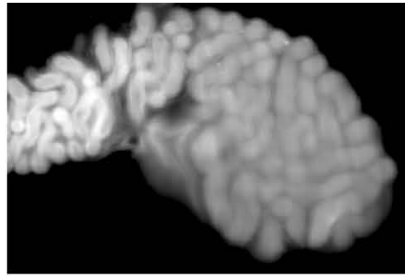

Cauda
Vas deferens
Caput

\section{Defb41iCre}

\section{Corpus}

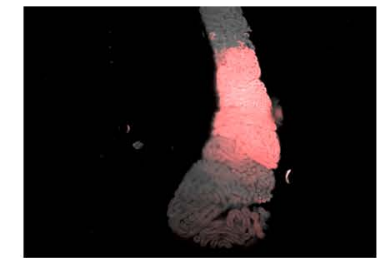

Caput

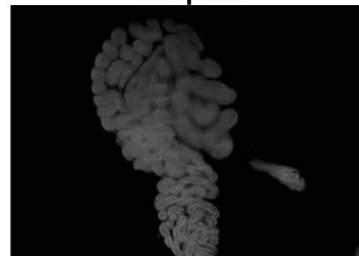

Cauda

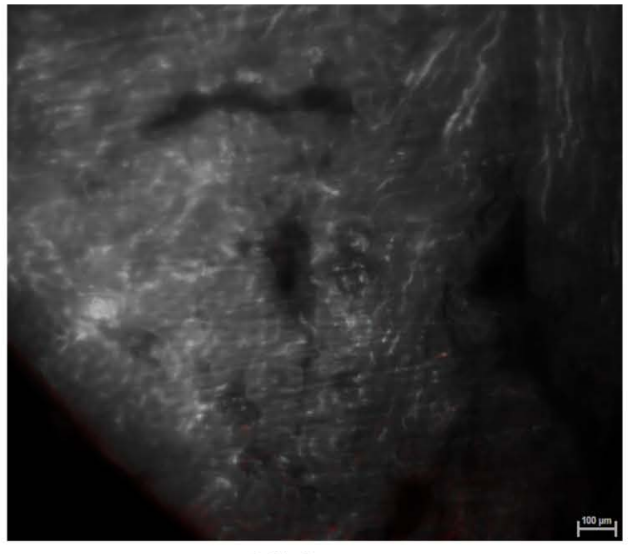

Kidney

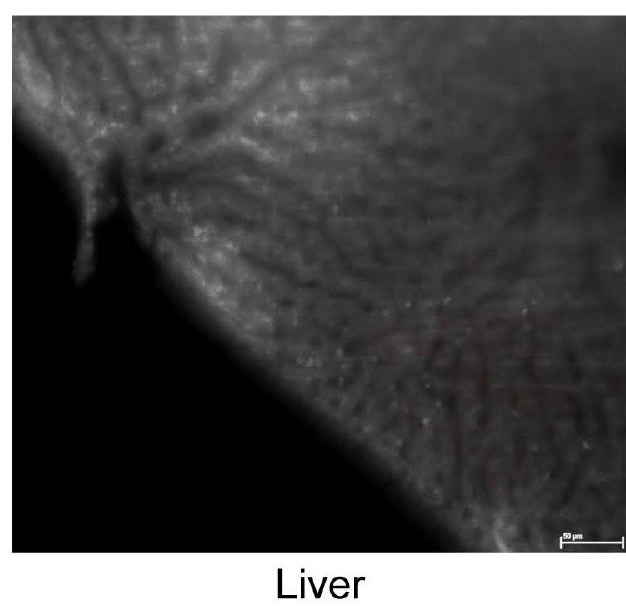


A

A B

AAV9-CMV-Cre

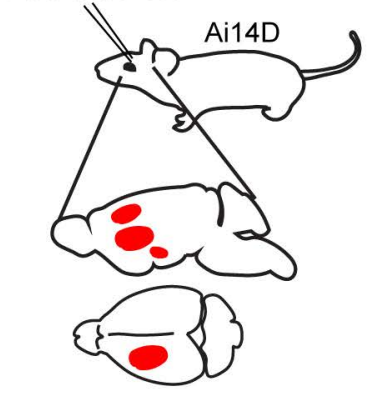

C

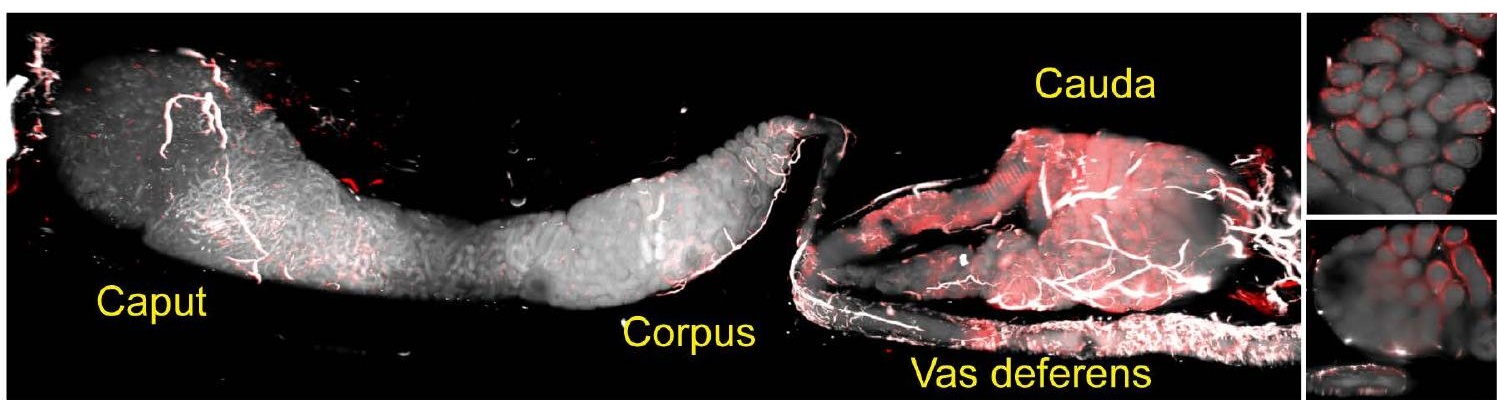

Figure 2

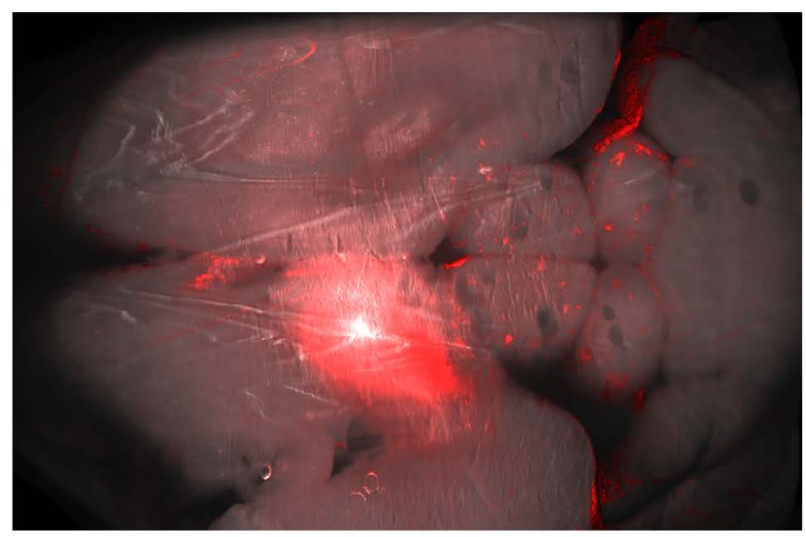


Figure 3

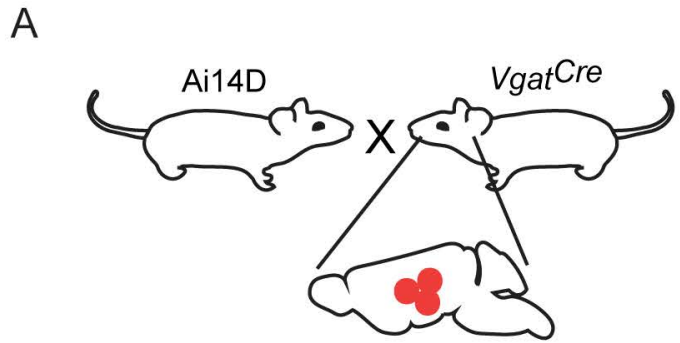

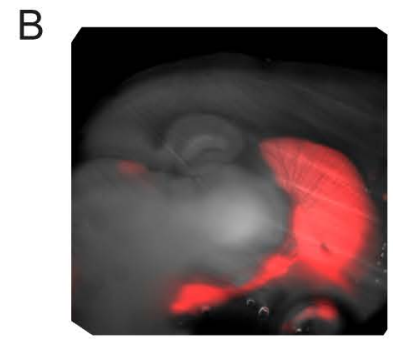

Brain

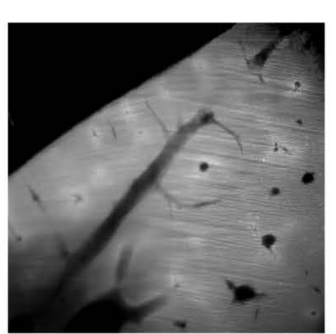

Liver

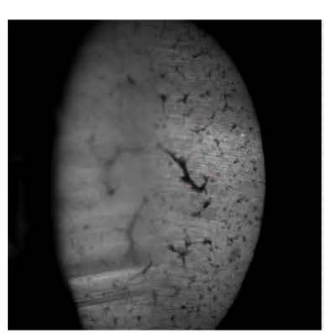

Kidney

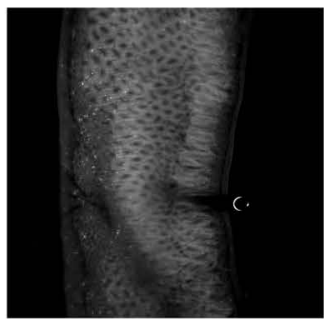

Intestine

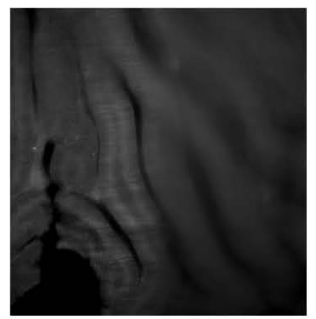

Testis

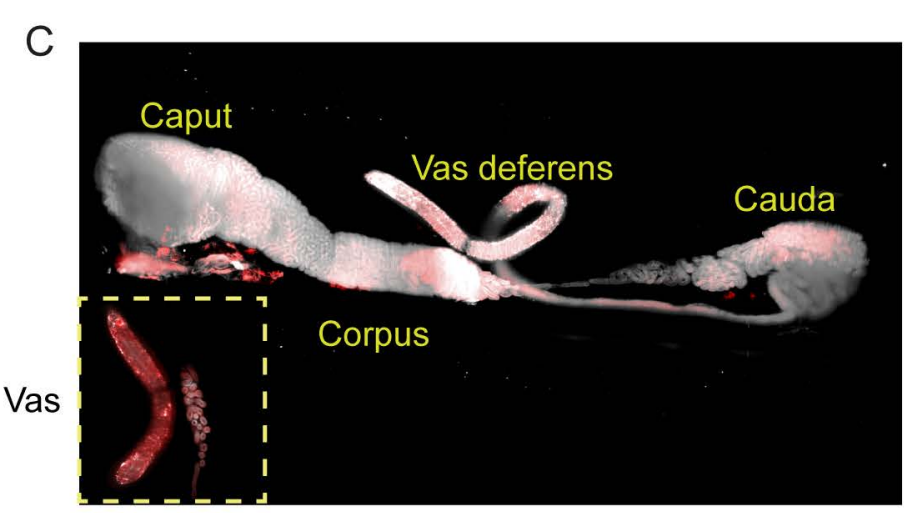

Ai14D; VgatCre

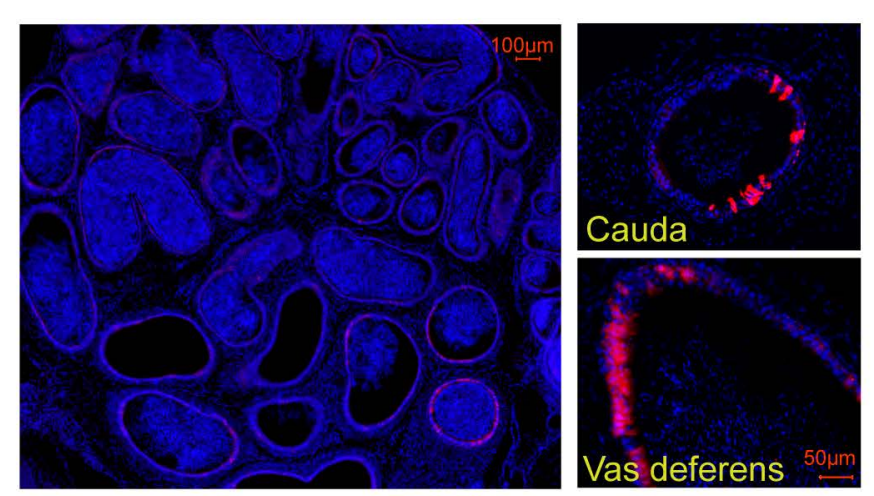

Cauda epididymis

E

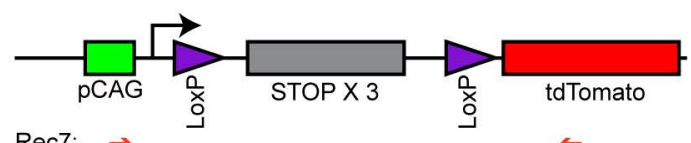

Rec7: $\rightarrow$

Rec11: $\rightarrow$

$\Sigma$

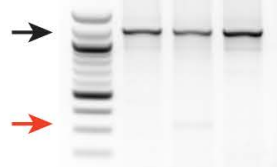

$-\infty+\infty=$

\begin{tabular}{|c|c|c|}
\hline \multicolumn{2}{|c|}{ No Cre } & \\
\hline
\end{tabular}

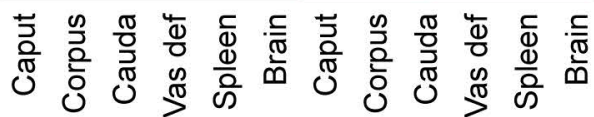

$\Sigma$

$\operatorname{Rec} 7$

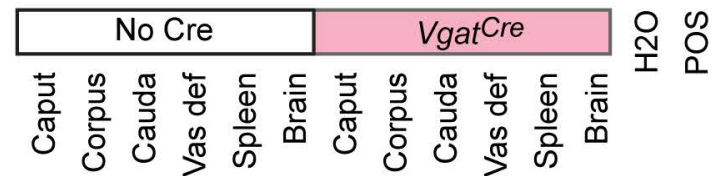


A

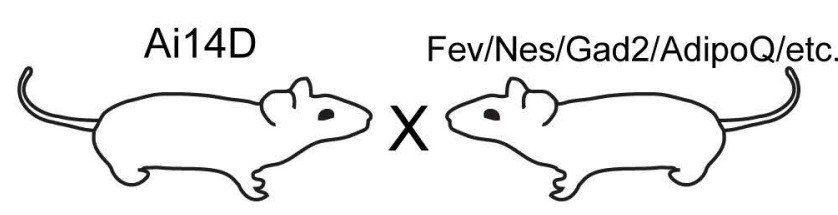

B

\section{Caput}

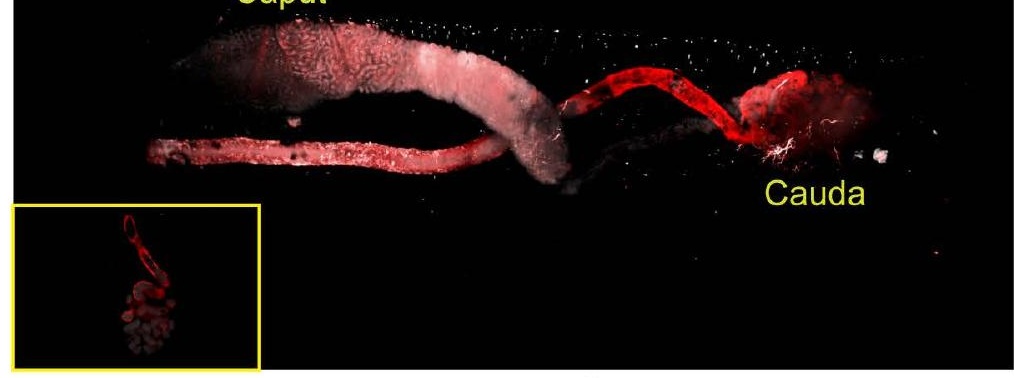

Ai14D; FevCre
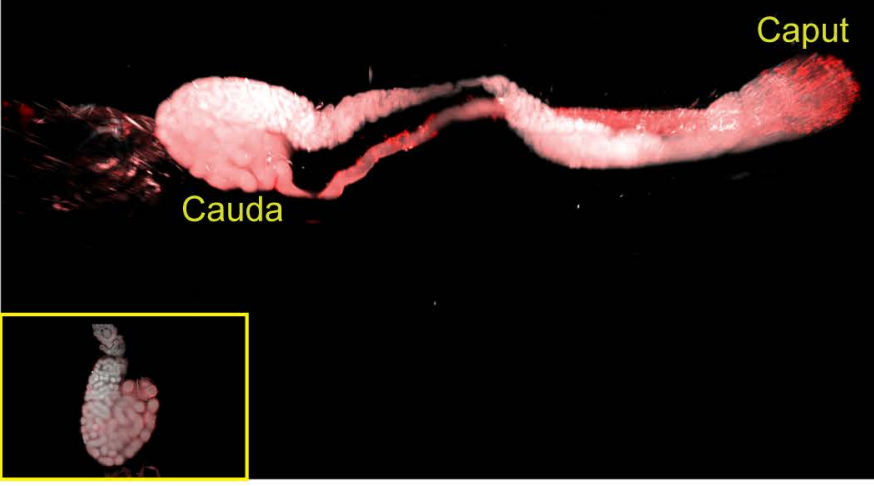

Ai14D; Drd1aCre

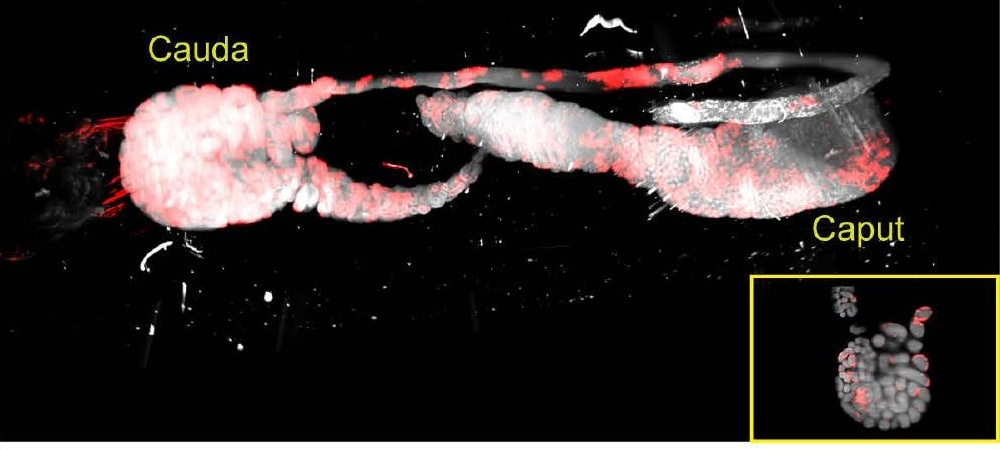


Figure 5

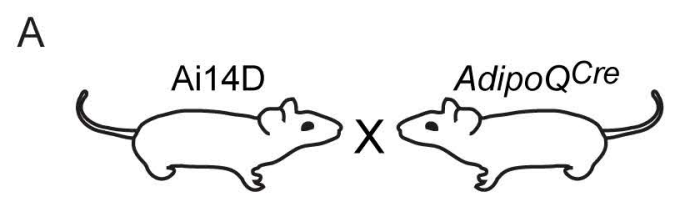

B

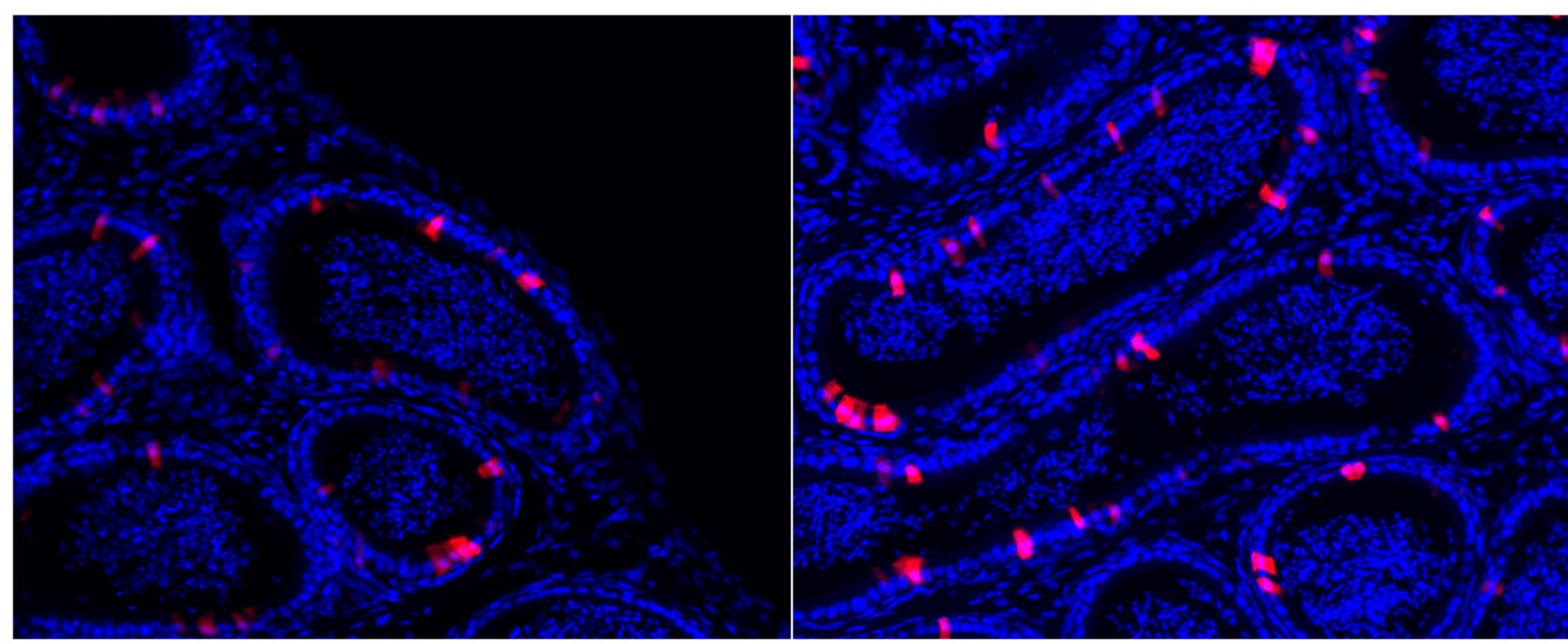

C

$\Sigma$

$\Sigma$

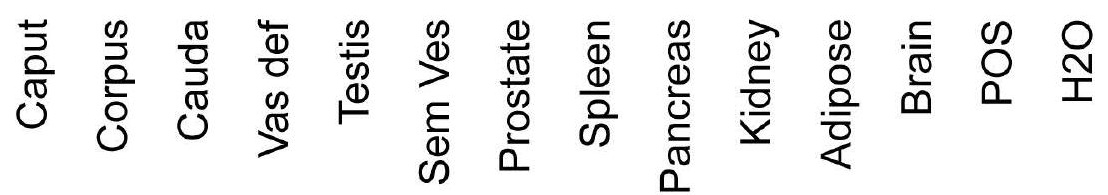


Figure S1

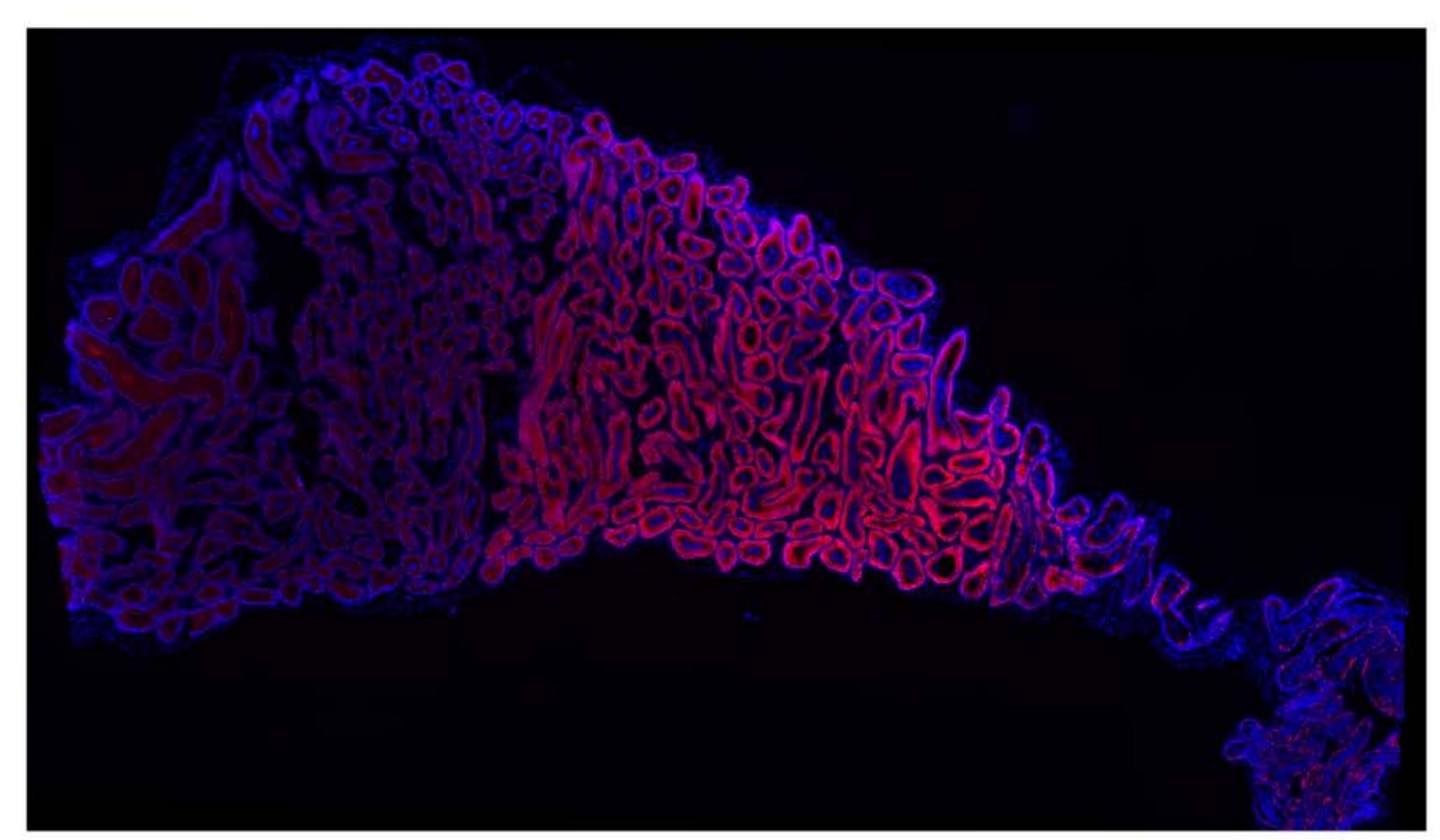

Ai14D; Defb41iCre

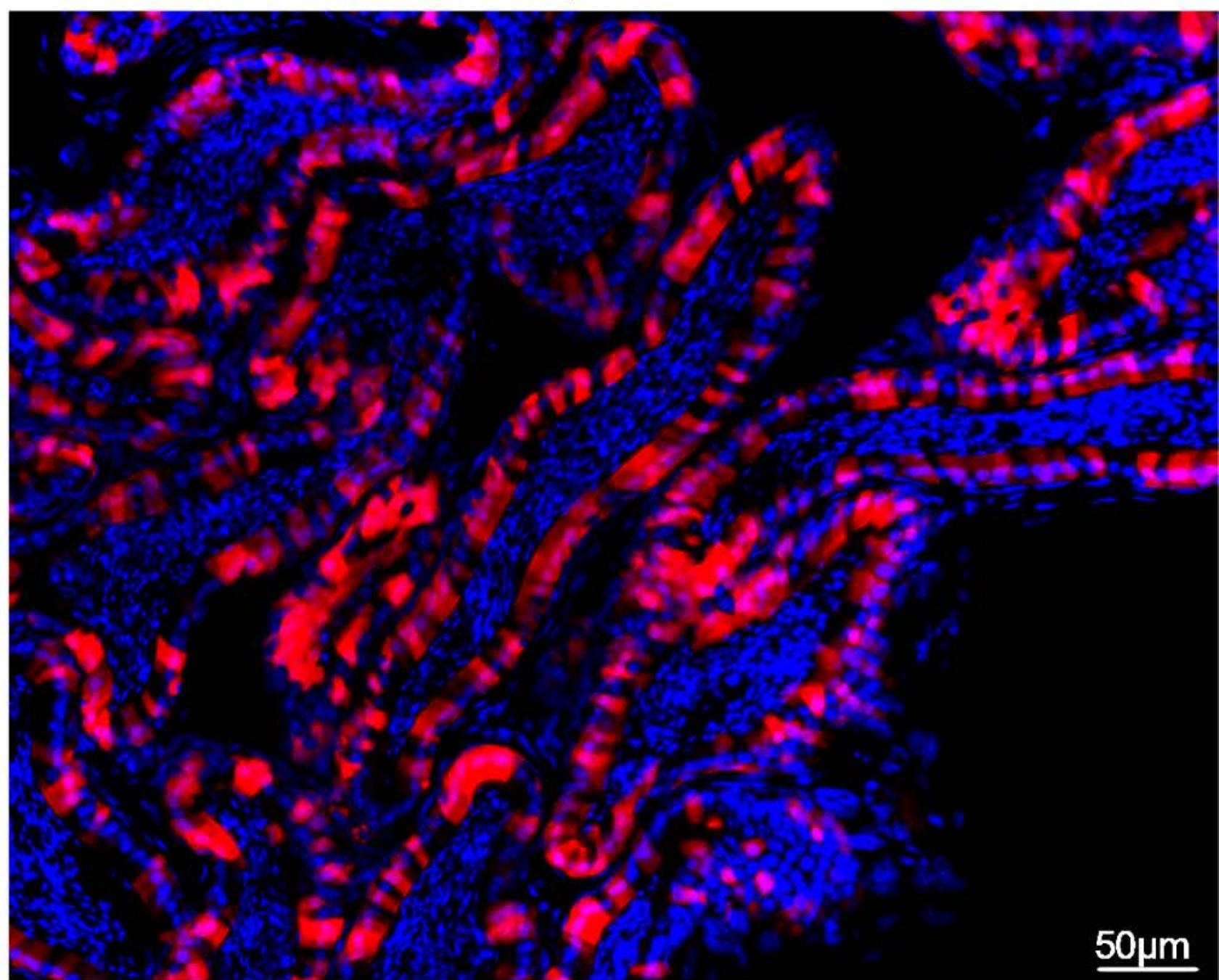


B
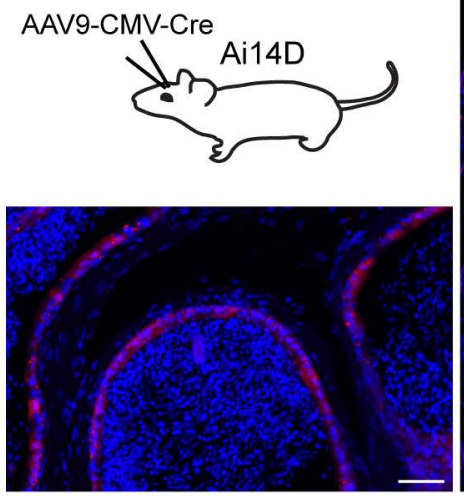

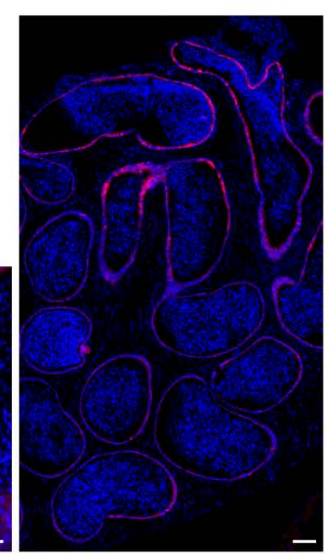

A
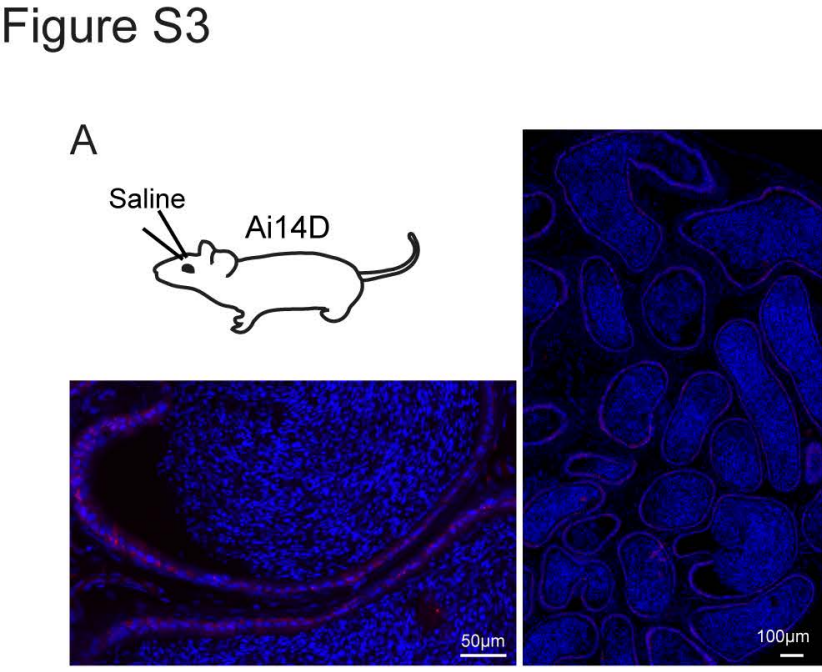
Figure S4

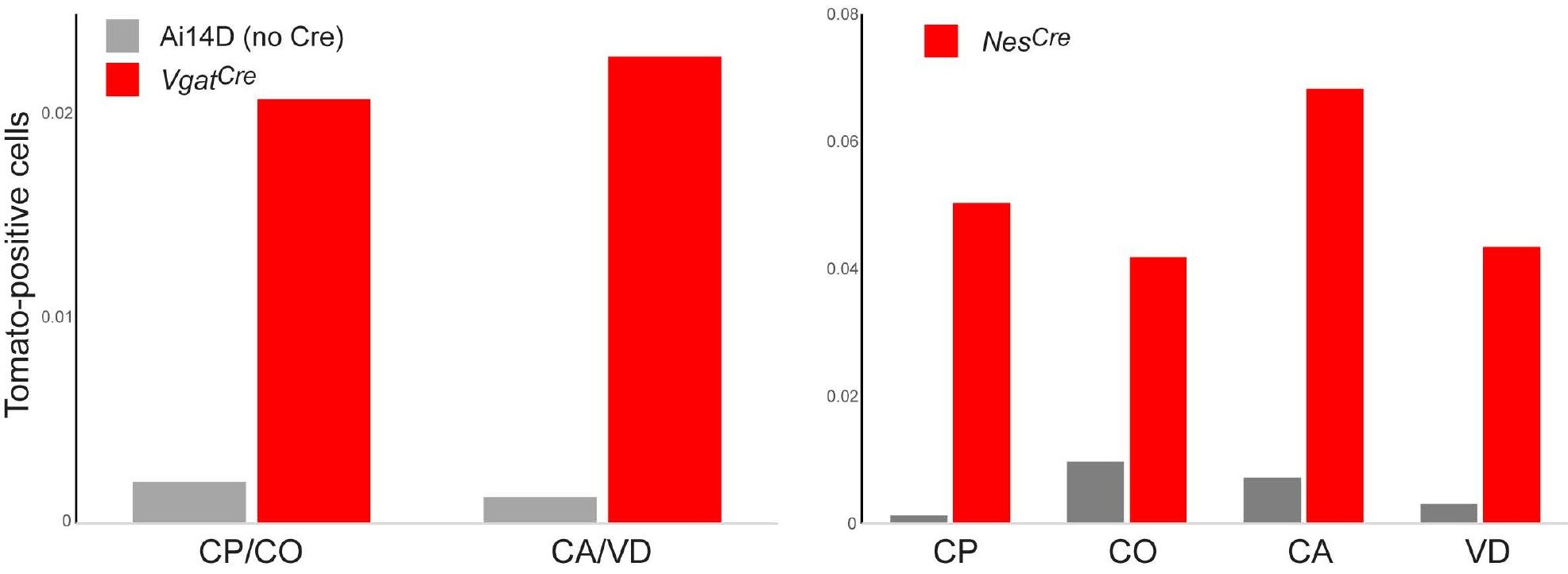


A

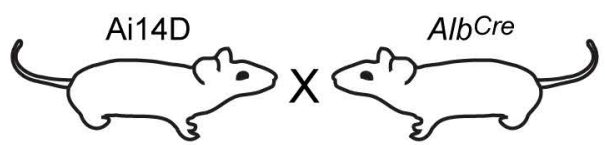

B

C

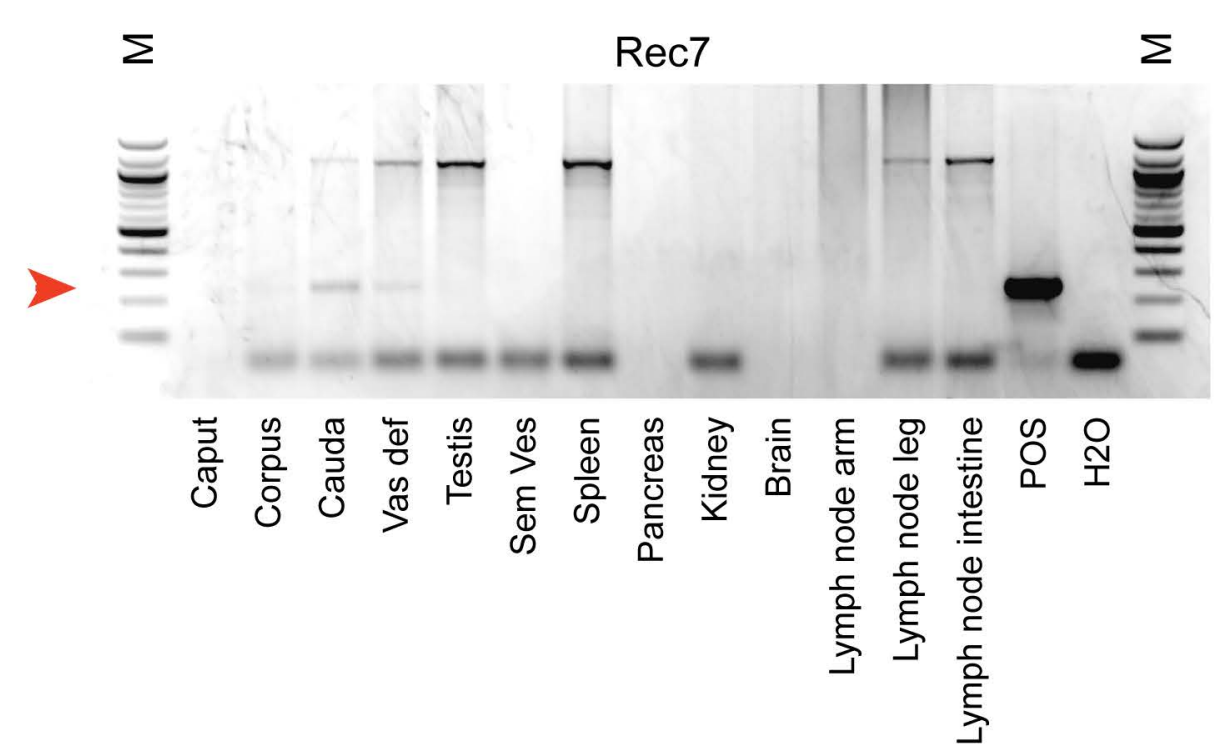


Ai14D; Syn1Cre

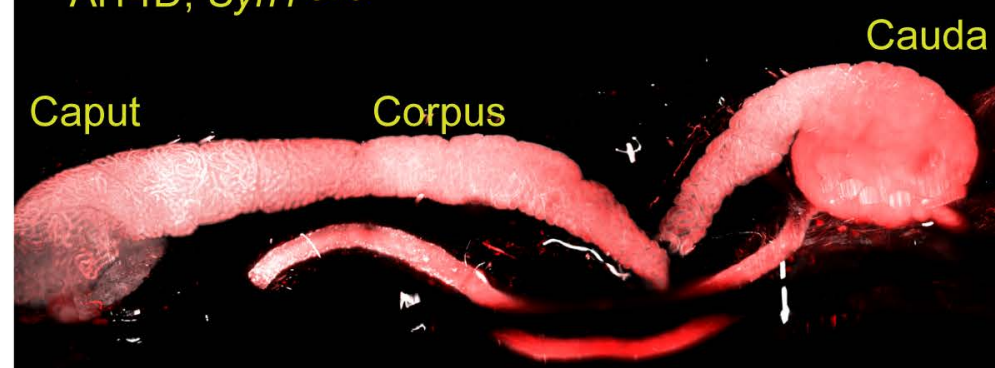

Vas deferens

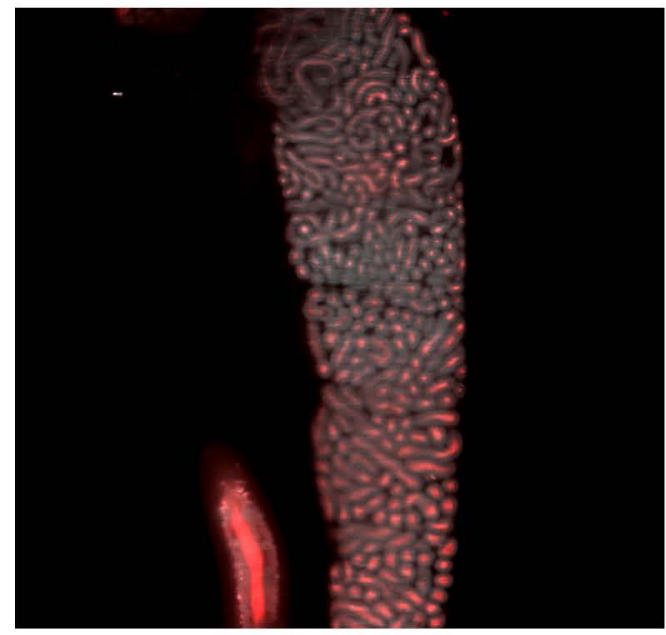

Caput

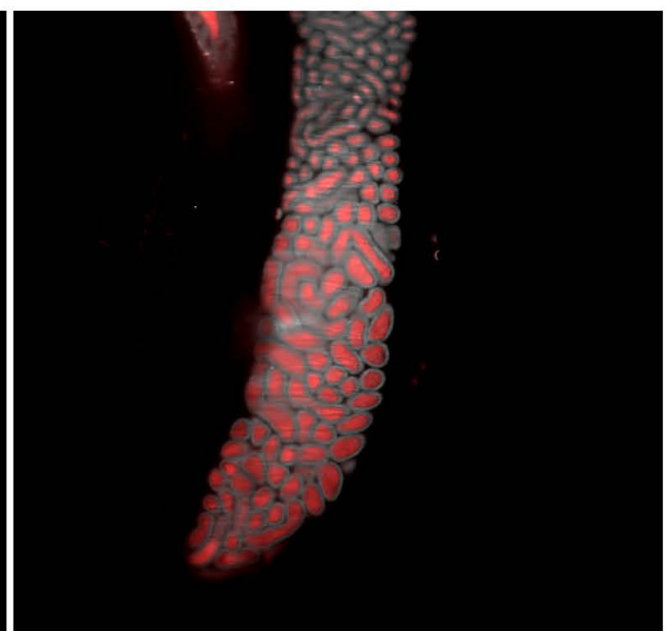

Corpus

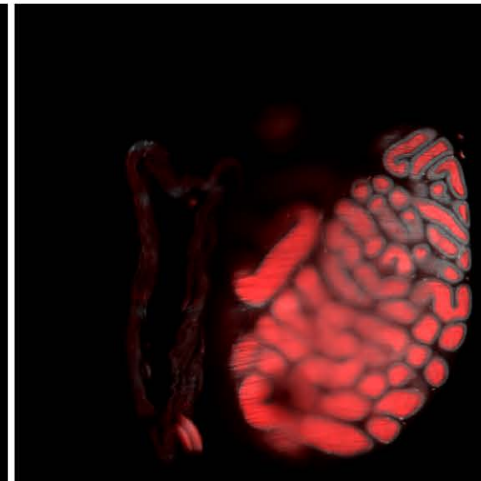

Cauda
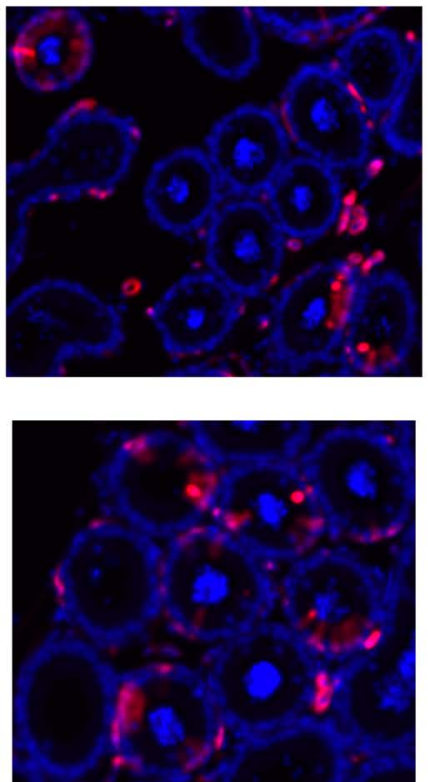


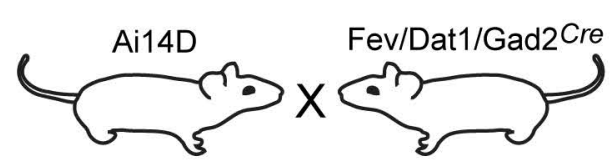

B

Kidney

Intestine

Testis

Accessory glands

Lung

Liver
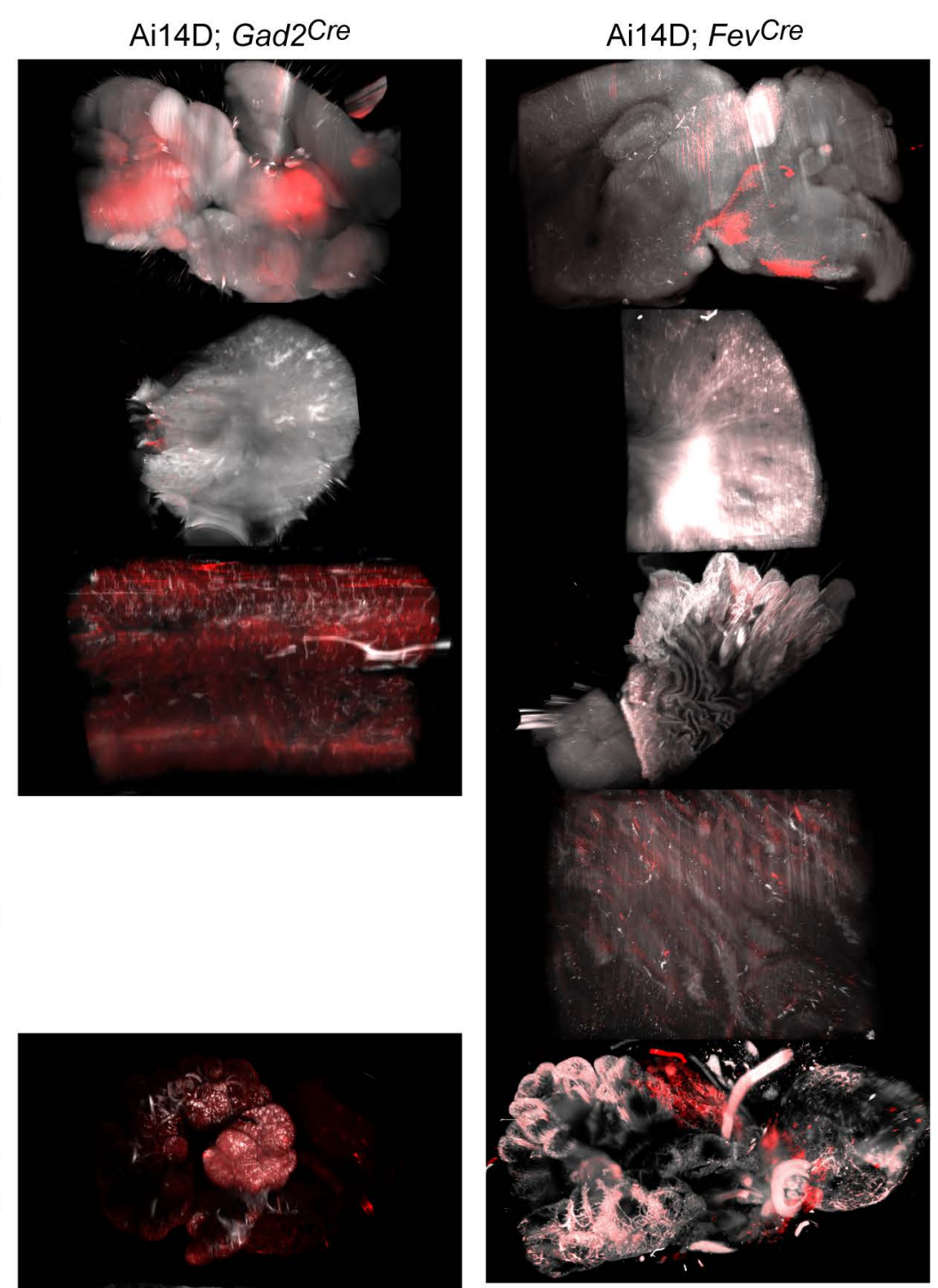

Spleen
Ai14D; Drd1aCre
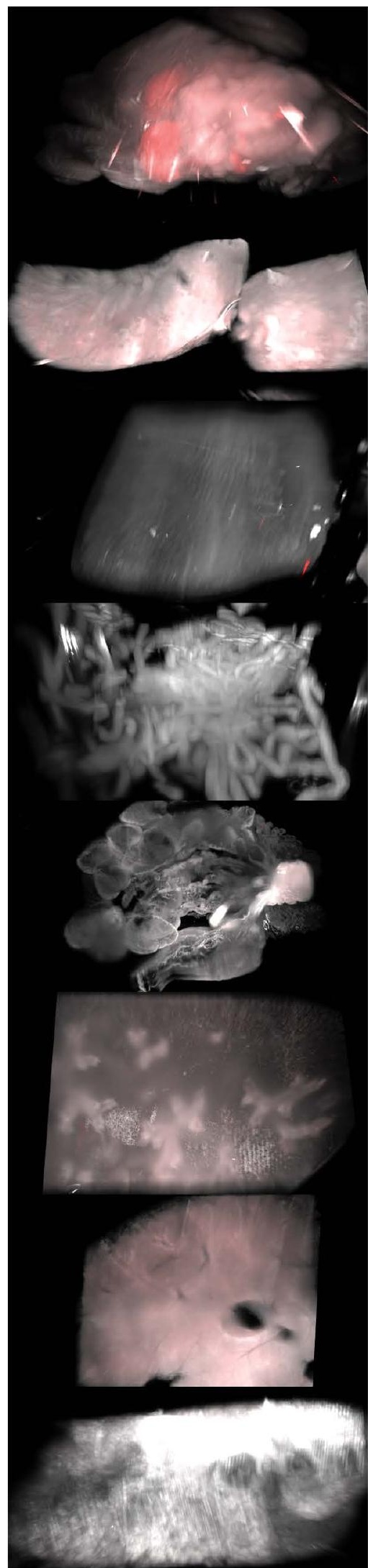
Figure S8

B

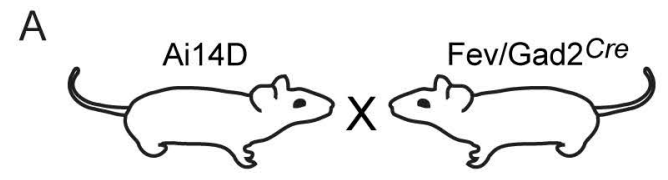

Accessory glands

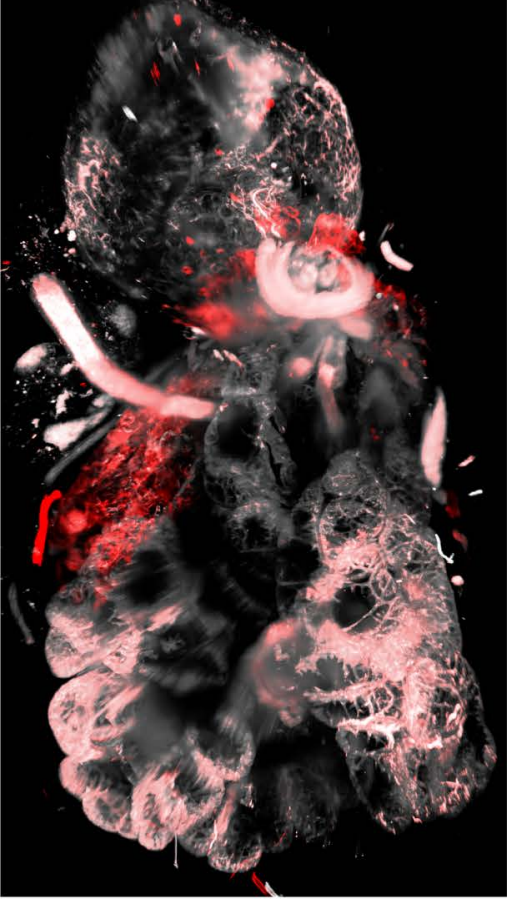

Ai14D; FevCre
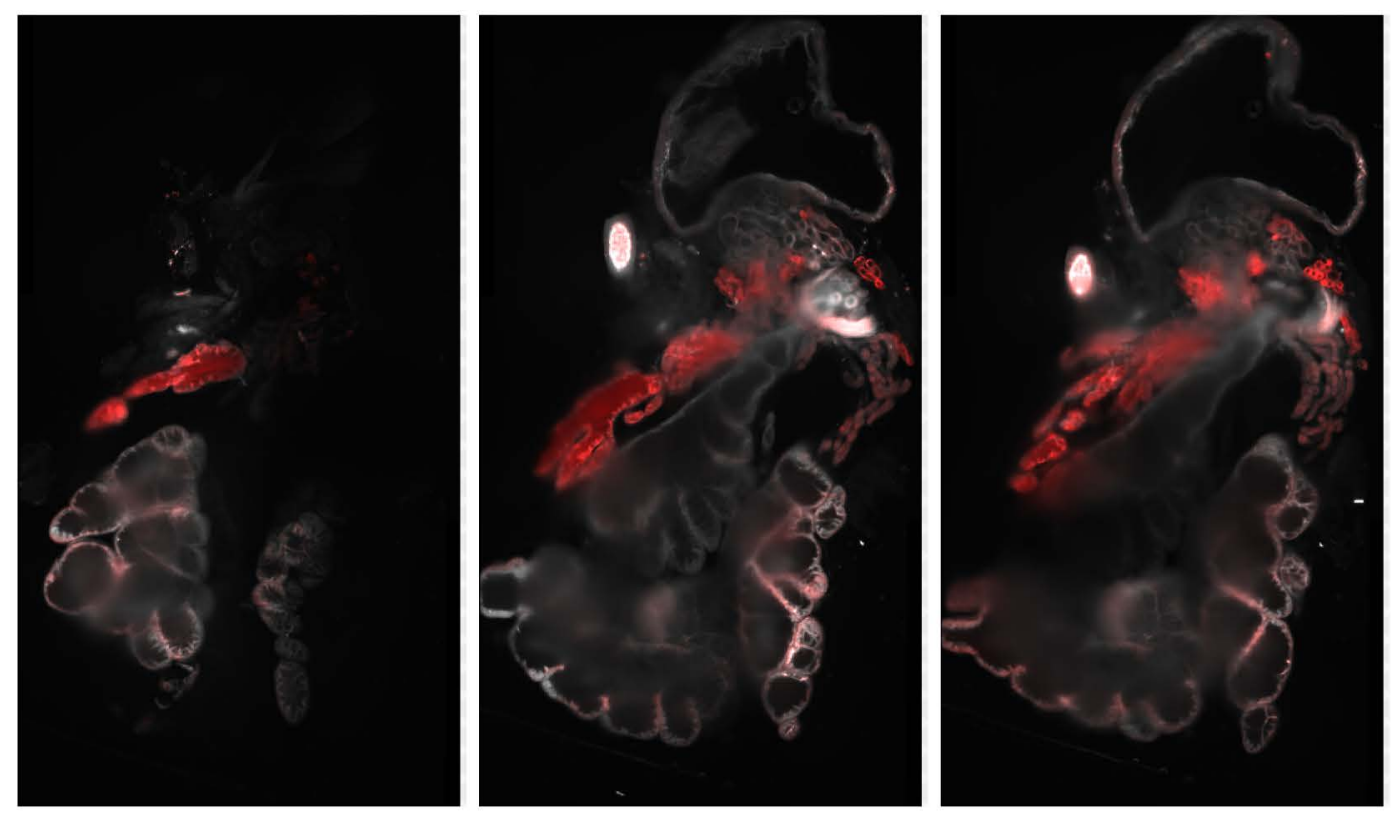
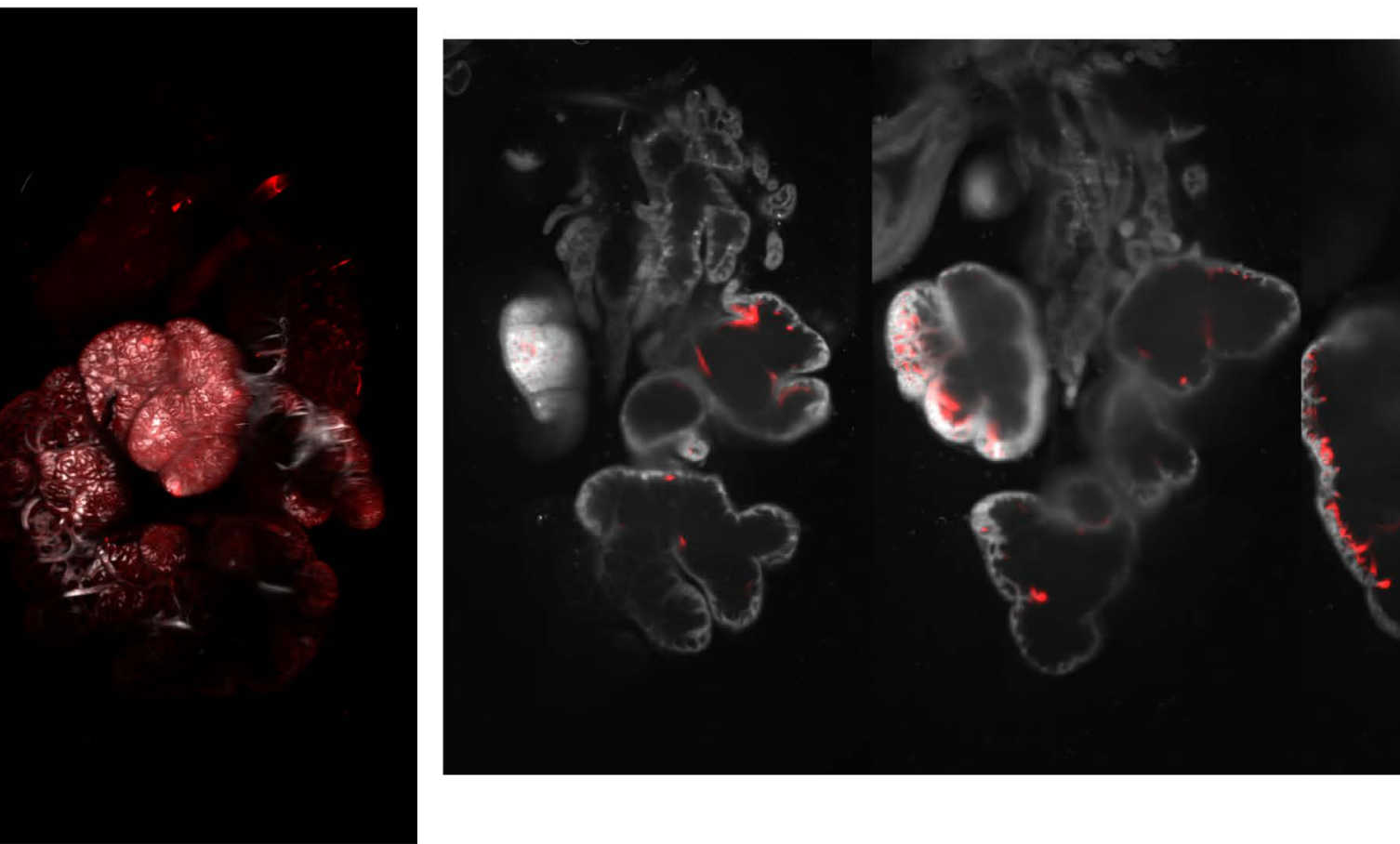
A

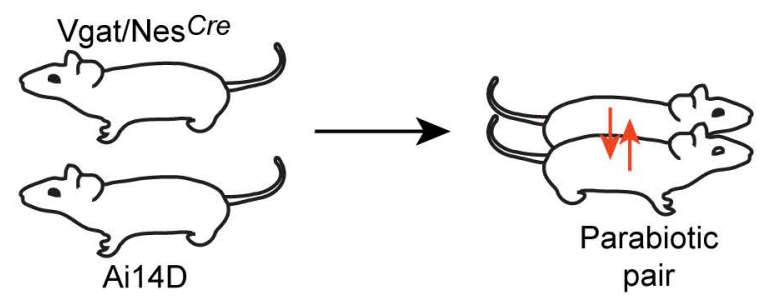

B

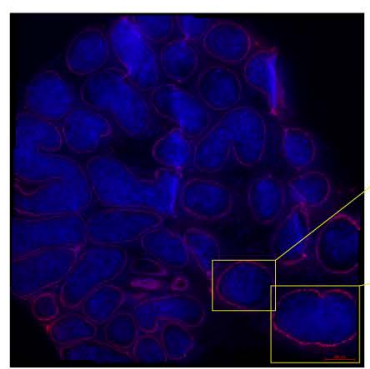

Ai14D:: Vgat Cre

NesCre

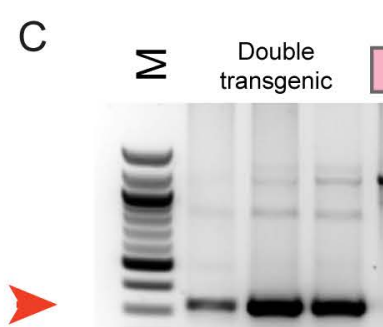

VgatCre

\begin{tabular}{l|l|l}
$\operatorname{Ai14D}$ & Cre & Ai14D \\
& & \\
& &
\end{tabular}

$\Sigma$

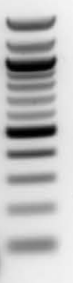

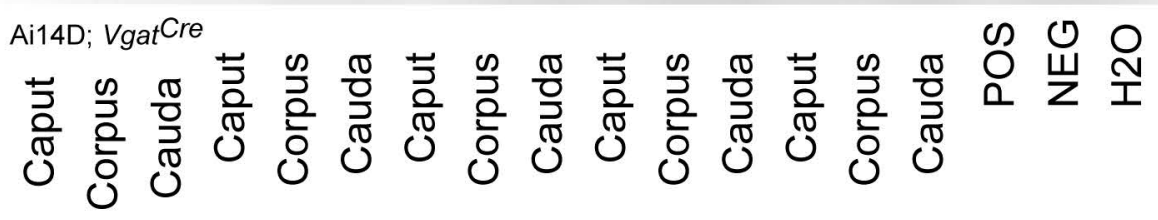

D

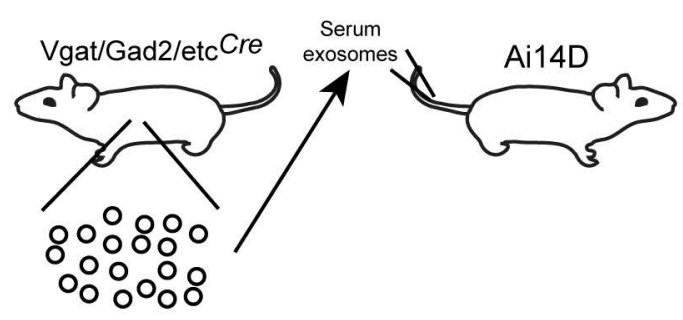

$\mathrm{F}$

Cauda epididymis, Rec11

$\Sigma \stackrel{\text { I }}{\text { I }}$

○

오 $\Sigma$

Serum transfer from:

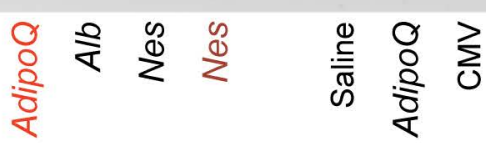

E
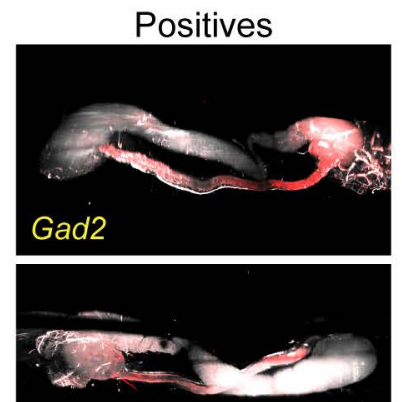

Gad2/Drd1a

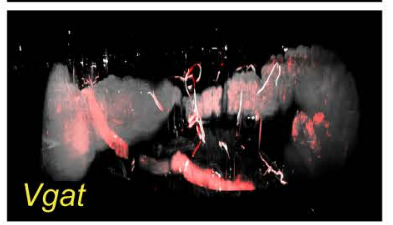

Negatives
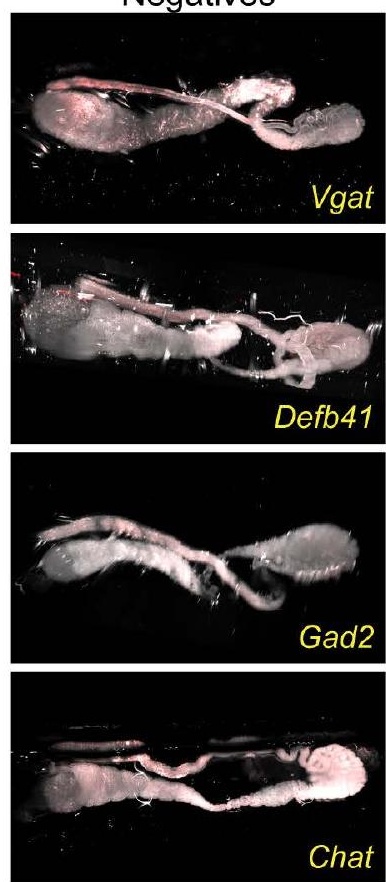

Chat

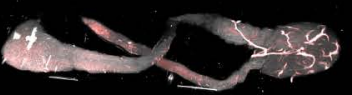




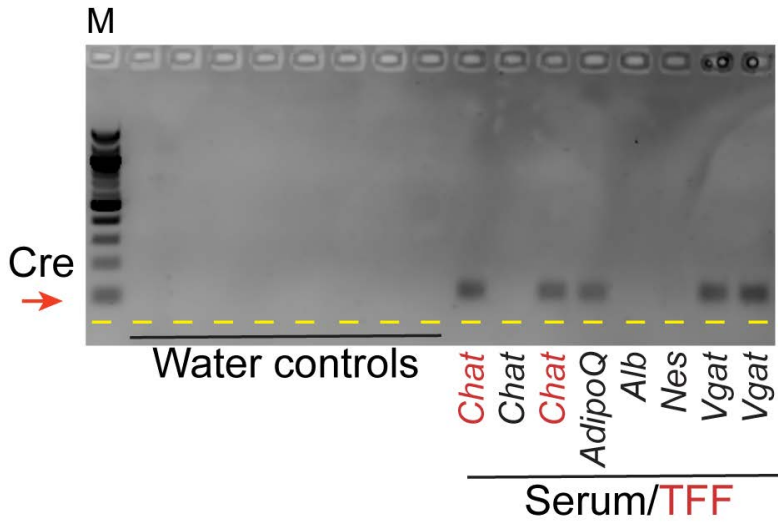

